\title{
COMPACTNESS PROPERTIES FOR TRACE-CLASS OPERATORS AND APPLICATIONS TO QUANTUM MECHANICS
}

\author{
J. DOLBEAULT, P. FELMER, AND J. MAYORGA-ZAMBRANO
}

\begin{abstract}
Interpolation inequalities of Gagliardo-Nirenberg type and compactness results for self-adjoint trace-class operators with finite kinetic energy are established. Applying these results to the minimization of various free energy functionals, we determine for instance stationary states of the Hartree problem with temperature corresponding to various statistics.
\end{abstract}

Key-words. Compact self-adjoint operators - Trace-class operators - mixed states - occupation numbers - Lieb-Thirring inequality - Gagliardo-Nirenberg inequality - logarithmic Sobolev inequality - optimal constants - orthonormal and sub-orthonormal systems Schrödinger operator - asymptotic distribution of eigenvalues - free energy - embeddings - compactness results

AMS MSC (2000). Primary: 81Q10, 82B10; Secondary: 26D15, 35J10, 47B34

\section{INTRODUCTION}

The first eigenvalue $\lambda_{V, 1}$ of a Schrödinger operator $-\Delta+V$ can be estimated using Sobolev's inequalities, $[24,22,11]$. In some recent papers, $[2,25,5]$, a precise connection has been given between the optimal estimates of $\lambda_{V, 1}$ in terms of a norm of $V$, and the optimal constants in some related Gagliardo-Nirenberg inequalities. Such inequalities admit optimal functions, see $[26,5]$. In the case of orthonormal and sub-orthonormal systems, interpolation inequalities of Gagliardo-Nirenberg type provide informations on optimal constants in inequalities, see $[16,15,9,8]$, which can be extended to Lieb-Thirring type inequalities, [14]. We refer to [5] for references in this direction and precise statements concerning the relation between optimal constants in these two families of inequalities, in the case of the euclidean space $\mathbb{R}^{d}$.

Conversely, the knowledge of Lieb-Thirring inequalities can be rephrased into interpolation inequalities for mixed states, which are infinite systems of orthogonal functions with occupations numbers, see [5]. It is well known that an equivalent formulation holds in terms of operators. In this paper we rewrite and extend these interpolation inequalities for trace-class self-adjoint operators and focus on the case

Date: July 7, 2006. 
of a domain $\Omega \subset \mathbb{R}^{d}$. We also study, at the level of the operators, the compactness properties of the corresponding embeddings, which somehow extend the well known properties of Sobolev's embeddings to trace-class self-adjoint operators.

An important source of motivation for us is the paper by Markowich, Rein and Wolansky, [18], which was devoted to the analysis of the stability of the Schrödinger-Poisson system. It involves in a crucial way some functionals which are a key tools of our approach, and that we call free energy functionals because of their interpretation in physics. In [18], the authors refer to such functionals as Casimir functionals, for historical reasons in mechanics, see for example [28]. During the last few years, various results based on free energy functionals, which are sometimes also called generalized entropy functionals, have been achieved in the theory of partial differential equations. We can for instance quote nonlinear stability results for fluid and kinetic equations, see for instance $[28,12,13,21]$, studies of the qualitative behavior of the solutions of kinetic and diffusion equations, including large time asymptotics and diffusion limits, see for example $[1,4,6]$, and applications to free boundary problems [7], or quantum mechanics [17, 18]. At a formal level, these various functionals are all more or less the same object, but the precise connection is still being studied at the moment from a mathematical point of view. However, at a physical the correspondence makes no more doubts.

Minimizing the free energy functional for a given potential is equivalent to proving Lieb-Thirring inequalities, while the optimization on the potential provides interpolation inequalities. Such questions have been only tangentially studied in [18], since in this paper the potential is an electrostatic Poisson potential with homogeneous Dirichlet boundary conditions and therefore always positive. Here we work in a much more general setting, which physically could correspond to external potentials with a singularity (for instance created by doping charged impurities in a semi-conductor) and our first task is therefore to bound from below the free energy functional, that is to establish adapted Lieb-Thirring inequalities. Our second step consists in reformulating these inequalities in terms of Gagliardo-Nirenberg type interpolation inequalities for operators, and to study the compactness properties of the corresponding embeddings. Afterwards, the minimization procedure becomes more or less trivial, thus giving for almost no work the existence of minimizers, including in the case of non-linear models involving, for instance, a Poisson coupling. 
Let $\Omega$ be a domain in $\mathbb{R}^{d}$ with smooth boundary and consider a smooth positive potential $V$ on $\bar{\Omega}$. As a starting point, we are interested in inequalities of Lieb-Thirring type for the Schrödinger operator $-\Delta+V$. Let $\left\{\lambda_{V, i}\right\}_{i \in \mathbb{N}^{*}}$ be the corresponding unbounded nondecreasing sequence of eigenvalues. As a straightforward consequence of the results of [5], the following inequality holds: for any $\gamma>d / 2$, there exists some explicit constant $C(\gamma)$, which does not depend on $V$, such that

$$
\sum_{i \in \mathbb{N}^{*}}\left(\lambda_{V, i}\right)^{-\gamma} \leq C(\gamma) \int_{\Omega} V^{d / 2-\gamma} d x
$$

(see Example 1 in Section 3.1 for a precise statement). This inequality arises as a special case of a "master inequality" which goes as follows. Consider a sequence of orthonormal functions $\left\{\psi_{i}\right\}_{i \in \mathbb{N}^{*}}$ and a non-increasing sequence $\left\{\nu_{i}\right\}_{i \in \mathbb{N}^{*}} \in \ell^{1}$ of non-negative real numbers. The sequence $\left\{\left(\nu_{i}, \psi_{i}\right)\right\}_{i \in \mathbb{N}^{*}}$ is called a mixed state in the physics literature. The master inequality is

$$
\sum_{i \in \mathbb{N}^{*}}\left[\beta\left(\nu_{i}\right)+\nu_{i}\left\langle\psi_{i},(-\Delta+V) \psi_{i}\right\rangle_{\mathrm{L}^{2}(\Omega)}\right] \geq-\sum_{i \in \mathbb{N}^{*}} F\left(\lambda_{V, i}\right) \geq-\int_{\Omega} G(V) d x .
$$

Here the functions $\beta, F$ and $G$ are related as follows. Consider a nonnegative function $g$ satisfying $\int_{0}^{\infty} g(t)\left(1+t^{-d / 2}\right) t^{-1} d t<\infty$, then we define

$$
F(s):=\int_{0}^{\infty} e^{-t s} g(t) \frac{d t}{t} \text { and } G(s):=\int_{0}^{\infty} e^{-t s}(4 \pi t)^{-d / 2} g(t) \frac{d t}{t},
$$

and we let $\beta$ be such that $\beta(s) \equiv F^{*}(-s)$, where $F^{*}$ denotes the Legendre-Fenchel transform of $F$. Then (1.1) corresponds to the case $\beta_{m}(\nu)=-c_{m} \nu^{m}$ for some explicit constant $c_{m}, m=\gamma /(\gamma+1) \in$ $(d /(d+2), 1), F(s) \equiv s^{-\gamma}$ and $G(s) \equiv C(\gamma) s^{d / 2-\gamma}$. The important point is that Inequality (1.2) holds for any positive potential and any mixed state. Other choices can also be taken, for instance $\beta_{1}(s) \equiv$ $s \log s-s, F(s) \equiv e^{-s}$ and $G(s) \equiv(4 \pi)^{-d / 2} e^{-s}$, thus showing the following Lieb-Thirring type inequality

$$
\sum_{i \in \mathbb{N}^{*}} e^{-\lambda_{V, i}} \leq(4 \pi)^{-d / 2} \int_{\Omega} e^{-V} d x .
$$

Using the Hilbert-Schmidt theorem, by considering self-adjoint traceclass operators $L$, with eigenpairs $\left\{\left(\nu_{i} \psi_{i}\right)\right\}$ and with kernel $K_{L}(x, y) \equiv$ $\sum_{i \in \mathbb{N}^{*}} \nu_{i} \psi_{i}(x) \psi_{i}(y)$, we can reformulate the first part of Inequality (1.2) 
in terms of operators:

$$
\begin{aligned}
& \mathcal{F}_{V, \beta}^{\lambda}(L) \equiv \sum_{i \in \mathbb{N}^{*}} {\left[\beta\left(\nu_{i}\right)+\nu_{i}\left\langle\psi_{i},(-\Delta+V-\lambda) \psi_{i}\right\rangle_{\mathrm{L}^{2}(\Omega)}\right] } \\
& \geq-\operatorname{Tr}[F(-\Delta+V-\lambda)]
\end{aligned}
$$

for some parameter $\lambda$ that we may take equal to 0 for the moment. Up to now, $V$ was assumed to be non-negative. Our first main result is an extension of Inequality (1.2) to potentials which may change sign, being possibly unbounded below. Assuming that $-(1-\varepsilon) \Delta+V-\lambda$ is a nonnegative operator for some $\varepsilon \in(0,1)$ and $\lambda \in \mathbb{R}$, for any non-negative perturbation $W$ of a sign changing potential $V$, Inequality (1.2) is replaced by

$$
\mathcal{F}_{V+W, \beta}^{\lambda}(L) \geq-\varepsilon^{-d / 2} \int_{\Omega} G(W) d x
$$

An optimization on $W$ then gives an interpolation inequality of Gagliardo-Nirenberg type. To give a precise statement, let us consider $F, G$ and $\beta$ as before and let $\tau$ be such that $G(s) \equiv \tau^{*}(-s)$. Here $G^{*}$ denotes the Legendre-Fenchel transform of $G$. We also use the notation $\rho_{L}$ for the non-negative function $\sum_{i \in \mathbb{N}^{*}} \nu_{i}\left|\psi_{i}\right|^{2} \in L^{1}(\Omega)$, using a mixed state representation $\left\{\left(\nu_{i}, \psi_{i}\right)\right\}_{i \in \mathbb{N}^{*}}$ associated to $L$. Some standard precautions are needed to identify $\rho_{L}(x)$ with $K_{L}(x, x)$.

Theorem 1.1. For a given potential $V$, assume that for some $\varepsilon \in$ $(0,1),-(1-\varepsilon) \Delta+V$ is bounded from below by some constant $\lambda$, in the sense of operators. With the above notations, Inequality (1.3) holds for any non-negative self-adjoint trace-class operator $L$, and moreover

$$
\mathcal{F}_{V, \beta}^{\lambda}(L) \geq \varepsilon^{-\frac{d}{2}} \int_{\Omega} \tau\left(\varepsilon^{\frac{d}{2}} \rho_{L}(x)\right) d x
$$

Two explicit important examples correspond to $F(s) \equiv s^{-\gamma}$ and $F(s) \equiv e^{-s}$. If we define the kinetic energy of a given trace-class operator $L$ as

$$
\mathcal{K}(L) \equiv \int_{\Omega} \nu_{i}|\nabla \psi|^{2} d x
$$

then we obtain the following interpolation inequalities:

$$
\mathcal{K}(L)+\kappa(\gamma) \int_{\Omega} \rho_{L}^{q} d x \geq c_{m} \operatorname{Tr}\left[L^{m}\right]
$$

where $q \equiv(2 \gamma-d) /(2(\gamma+1)-d) \in(0,1), \gamma>d / 2, m=\gamma /(\gamma+1)$ and $\kappa(\gamma)$ is an explicit positive constant, and

$$
\int_{\Omega} \rho_{L} \log \rho_{L} d x \leq \operatorname{Tr}[L \log L]+\frac{d}{2} \log \left(\frac{e}{2 \pi d} \frac{\mathcal{K}(L)}{\|L\|_{1}}\right)\|L\|_{1},
$$


where $L$ is any non-negative self-adjoint trace-class operator and $\|L\|_{1}$ denotes $\operatorname{Tr}[L]$. For simplicity, the inequalities written here correspond to the case where $V$ is non-negative, but more general statements corresponding to a sign changing potential $V$ can be deduced from Theorem 1.1.

The interpolation inequalities of Theorem 1.1 generalize for selfadjoint trace-class operators the usual Gagliardo-Nirenberg inequalities. Exactly as for the embedding $\mathrm{H}_{0}^{1}(\Omega) \hookrightarrow \mathrm{L}^{2}(\Omega)$, some compactness can be expected. Such a statement constitutes our second main result.

Theorem 1.2. Under the assumptions of Theorem 1.1, if $\left\{L_{n}\right\}_{n \in \mathbb{N}}$ is a sequence of non-negative self-adjoint trace-class operators such that

$$
\left\{\mathcal{F}_{V, \beta}^{\lambda}\left(L_{n}\right)\right\}_{n \in \mathbb{N}}
$$

is bounded, then $\left\{L_{n}\right\}_{n \in \mathbb{N}}$ is relatively compact and converges to a nonnegative self-adjoint compact operator $L$ up to a subsequence. Moreover, $\rho_{L_{n}}$ converges to $\rho_{L}$ in $L^{q}(\Omega)$, for any $q \in[1, \infty]$ if $d=1$, $q \in[1, \infty)$ if $d=2$ and $q \in[1, d /(d-2)]$ if $d \geq 3$.

See Theorem 3.3 for a precise notion of convergence of $\left\{L_{n}\right\}_{n \in \mathbb{N}}$.

This paper is organized as follows. Section 2 is devoted to definitions and preliminary results. In Section 2.1 we introduce the operator setting. In Section 2.2 we define a set of trace-class operators having the form $F(-\Delta)$. To this class belong the operators generated by the Boltzmann distribution and the Fermi-Dirac statistics, see Example 3 in Section 2.2. The space $\mathcal{S}_{1}$ of trace-class self-adjoint operators, which are also known as nuclear self-adjoint operators, plays the role of the space $\mathrm{L}^{1}$ and the spaces $\mathcal{S}_{q}$ can be felt as a generalization of the spaces $\mathrm{L}^{q}, q \in[1, \infty]$. Inspired by this analogy, we define in Section 2.3 the Sobolev-like cones $\mathcal{W}^{l, p}$ as appropriate subsets of $\mathcal{S}_{1}$. As far as we know the definition of these cones is a novelty. Basic properties (Proposition 2.1) of these cones and a regularity result (Proposition 2.2) concerning the density functions associated to $\mathcal{H}^{1}=\mathcal{W}^{1,2}$ are established in Section 2.3. The free energy functional $\mathcal{F}_{V, \beta}^{\lambda}(L)$ is defined in Section 2.4.

Theorems 1.1 and 1.2 are proved in Section 3. An improved interpolation inequality is given in Theorem 3.2. The key estimate is a convexity inequality (Lemma 3.1) which allows simultaneously to minimize the free energy functional and to get some coercivity even if $V$ changes sign (Proposition 3.5). The compactness result then follows (see Theorem 3.3 for a detailed statement.)

As a simple consequence, in Section 4, we prove the existence of minimizers in several cases of interest in quantum mechanics. Some 
additional references for applications in quantum mechanics are given at the end of this paper.

\section{Definitions AND PRELIMINARY RESUlts}

2.1. The operators setting. Let $\Omega$ be a domain in $\mathbb{R}^{d}, d \in \mathbb{N}^{*}$. We denote by $\mathcal{L}\left(\mathrm{L}^{2}(\Omega)\right)$ the space of bounded linear operators acting on $\mathrm{L}^{2}(\Omega)$ and by $\|\cdot\|$ its standard norm. In $\mathcal{L}\left(\mathrm{L}^{2}(\Omega)\right)$ we consider the subspaces $\mathcal{I}_{\infty}$ and $\mathcal{S}_{\infty}$ of compact and compact self-adjoint operators, respectively. Next we define the space of trace-class operators which is a subspace of $\mathcal{I}_{\infty}$ given by

$$
\mathcal{I}_{1} \equiv\left\{L \in \mathcal{I}_{\infty}: \sum_{i \in \mathbb{N}^{*}}\left|\left\langle\chi_{i}, L \chi_{i}\right\rangle_{\mathrm{L}^{2}(\Omega)}\right|<\infty\right\}
$$

where $\left\{\chi_{i}\right\}_{i \in \mathbb{N}^{*}}$ is any complete orthonormal system in $\mathrm{L}^{2}(\Omega)$. The elements of $\mathcal{I}_{1}$ are indifferently called trace-class operators or nuclear operators. Given $L \in \mathcal{I}_{1}$, the trace of $L$ is the value

$$
\operatorname{Tr}[L] \equiv \sum_{i \in \mathbb{N}^{*}}\left\langle\chi_{i}, L \chi_{i}\right\rangle_{\mathrm{L}^{2}(\Omega)}
$$

where $\left\{\chi_{i}\right\}_{i \in \mathbb{N}^{*}}$ is any complete orthonormal system in $\mathrm{L}^{2}(\Omega)$. It is a well known fact of the theory that the trace of an operator does not depend on the choice of $\left\{\chi_{i}\right\}_{i \in \mathbb{N}^{*}}$. We will also consider the space of the Hilbert-Schmidt operators, which is defined as

$$
\mathcal{I}_{2} \equiv\left\{L \in \mathcal{I}_{\infty}: \sum_{i \in \mathbb{N}^{*}}\left|\left\langle\chi_{i},|L|^{2} \chi_{i}\right\rangle_{\mathrm{L}^{2}(\Omega)}\right|<\infty\right\} .
$$

Equipped with the scalar product $\langle L, R\rangle_{2} \equiv \operatorname{Tr}\left[R^{*} L\right], \mathcal{I}_{2}$ is a Hilbert space. We denote the corresponding norm by $\|\cdot\|_{2}$. It can be proved, see for instance [19, Theorem VI.23], that an operator $L \in \mathcal{L}\left(\mathrm{L}^{2}(\Omega)\right)$ belongs to the Hilbert-Schmidt space if and only if there is a function $K_{L} \in \mathrm{L}^{2}(\Omega \times \Omega)$, the kernel of $L$, such that

$$
\|L\|_{2}^{2}=\iint_{\Omega \times \Omega}\left|K_{L}(x, y)\right|^{2} d x d y
$$

and

$$
(L \eta)(x)=\int_{\Omega} K_{L}(x, y) \eta(y) d y \quad \text { for } x \in \Omega \text { a.e. }, \quad \forall \eta \in \mathrm{L}^{2}(\Omega) .
$$

For $L \in \mathcal{S}_{\infty}$ we denote by $\left\{\nu_{i}(L)\right\}_{i \in \mathbb{N}^{*}}$, or simply $\left\{\nu_{i}\right\}_{i \in \mathbb{N}^{*}}$ if there is no confusion, the sequence of eigenvalues of $L$ counted with multiplicity, which is well defined by the Hilbert-Schmidt theorem. We adopt the convention that $\left\{\nu_{i}\right\}_{i \in \mathbb{N}^{*}}$ is ordered in a way such that $\left\{\left|\nu_{i}\right|\right\}_{i \in \mathbb{N}^{*}}$ is nonincreasing, and if both $\nu$ and $-\nu$ are eigenvalues, $-|\nu|$ comes first. We 
will denote by $\left\{\psi_{i}(L)\right\}$, or simply $\left\{\psi_{i}\right\}_{i \in \mathbb{N}^{*}}$ if there is no ambiguity, an associated orthonormal system of eigenfunctions, which is complete in $\mathrm{L}^{2}(\Omega)$ : see, e.g., [3, Chapter VI]. From now on we are only dealing with self-adjoint operators and consider for any $q \in[1, \infty)$ the spaces

$$
\mathcal{S}_{q} \equiv\left\{L \in \mathcal{S}_{\infty}:\|L\|_{q} \equiv\left(\sum_{i \in \mathbb{N}^{*}}\left|\nu_{i}\right|^{q}\right)^{1 / q}<\infty\right\}
$$

If $L \in \mathcal{S}_{2}$, given an orthonormal basis of $L^{2}(\Omega)$ of eigenfunctions $\left\{\psi_{i}\right\}_{i \in \mathbb{N}^{*}}$ associated to $L, K_{L}$ is explicitly given by

$$
K_{L}(x, y)=\sum_{i \in \mathbb{N}^{*}} \nu_{i} \psi_{i}(x) \bar{\psi}_{i}(y) \quad \text { for } x, y \in \Omega \text { a.e., }
$$

and the two given definitions of $\|L\|_{2}$ coincide.

If $L \in \mathcal{S}_{1}$ and $\left\{\psi_{i}\right\}_{i \in \mathbb{N}^{*}}$ is an orthonormal basis of $L^{2}(\Omega)$ of eigenfunctions associated to $L \in \mathcal{S}_{1}$, we define

$$
\rho_{L}(x) \equiv \sum_{i \in \mathbb{N}^{*}} \nu_{i}\left|\psi_{i}(x)\right|^{2} \quad x \in \Omega \text { a.e. },
$$

which is in $\mathrm{L}^{1}(\Omega)$. It is a well known fact that $\rho_{L}$ does not depend on the special choice of $\left\{\psi_{i}\right\}_{i \in \mathbb{N}^{*}}$ and that

$$
\int_{\Omega}\left|\rho_{L}(x)\right| d x \leq\|L\|_{1}=\operatorname{Tr}(|L|)=\int_{\Omega} \rho_{|L|}(x) d x \quad \forall L \in \mathcal{S}_{1} .
$$

If additionally $L$ is a non-negative operator, $\rho_{L}$ is also non-negative and it is called the density function associated to $L$. We certainly have $\|L\|_{1}=\operatorname{Tr}[L]=\int_{\Omega} \rho_{L}(x) d x$. Such a definition is consistent with the density operator formalism in quantum mechanics.

Remark 2.1. In some cases (2.1) makes sense for an operator $L$ which is not in $\mathcal{S}_{1}$, but is for instance in $\mathcal{L}\left(\mathrm{L}^{2}(\Omega)\right)$ and such that the right hand side in (2.1) is finite. We shall then write $\operatorname{tr}[L]$ instead of $\operatorname{Tr}[L]$.

Let us recall some other well known facts on $\mathcal{S}_{q}$. We refer the reader to [20, Prop. 5-6] for more details.

i) $\mathcal{S}_{q}$ equipped with the norm $\|\cdot\|_{q}$ is a Banach space and $\|L\|=$ $\lim _{q \rightarrow \infty}\|L\|_{q}$, but $\mathcal{S}_{q} \subsetneq \mathcal{S}_{\infty}$ for any $q \in[1, \infty)$.

ii) If $1<q_{1}<q_{2}<\infty$, then

$$
\|L\|_{q_{2}} \leq\|L\|_{q_{1}} \quad \forall L \in \mathcal{S}_{q_{1}},
$$

so that $\mathcal{S}_{1} \subset \mathcal{S}_{q_{1}} \subset \mathcal{S}_{q_{2}} \subset \mathcal{S}_{\infty}$.

iii) $\mathcal{S}_{q}$ is the closure of the space of finite rank self-adjoint operators with respect to the norm $\|\cdot\|_{q}$. 
iv) If $1 \leq q \leq \infty$ and $q^{-1}+r^{-1}=1$, then

$$
\|A B\|_{1} \leq\|A\|_{q}\|B\|_{r} \quad \forall A \in \mathcal{S}_{q}, B \in \mathcal{S}_{r} .
$$

In case $q=\infty$ (and $r=1),\|\cdot\|_{\infty}=\|\cdot\|$ is the usual norm of bounded operators.

\subsection{Operators of the form $\mathbf{F}(-\boldsymbol{\Delta})$ and Casimir-type functions.}

In the case of a bounded domain $\Omega \subset \mathbb{R}^{d}$, a useful class of operators can be obtained out of the Laplacian. Let $\left\{\lambda_{0, i}\right\}_{i \in \mathbb{N}^{*}}$ and $\left\{\phi_{0, i}\right\}_{i \in \mathbb{N}^{*}}$ be the eigenvalues and eigenfunctions of the Laplacian with homogeneous Dirichlet boundary conditions, that is for each $i \in \mathbb{N}^{*}$

$$
-\Delta \phi_{0, i}=\lambda_{0, i} \phi_{0, i} \quad \text { in } \Omega, \quad \phi_{0, i} \in \mathrm{H}_{0}^{1}(\Omega) .
$$

The ordered sequence $0<\lambda_{0,1}<\lambda_{0,2} \leq \lambda_{0,3} \leq \ldots$ diverges and $\left\{\phi_{0, i}\right\}_{i \in \mathbb{N}^{*}}$ is a complete orthonormal system in $\mathrm{L}^{2}(\Omega)$. Moreover, $\phi_{0, i} \in$ $\mathrm{C}^{\infty}(\Omega)$ for all $i \in \mathbb{N}^{*}$. See for instance, [3, Theorem IX.31],

Definition 2.1. We shall say that a function $F: \mathbb{R} \rightarrow \mathbb{R} \cup\{+\infty\}$ is of class $\mathcal{C}(-\Delta, \Omega)$ if $F$ is convex and $\sum_{i \in \mathbb{N}^{*}} F\left(\lambda_{0, i}\right)$ is finite.

The Spectral Theorem (see for instance [19, Theorem VIII.5]) then allows to define the trace-class operator $F(-\Delta)$ for each $F \in \mathcal{C}(-\Delta, \Omega)$. We observe that in this case it follows that the spectrum $\sigma(-\Delta) \equiv$ $\left\{\lambda_{0, i}: i \in \mathbb{N}^{*}\right\}$ of $-\Delta$ is contained in the domain $\operatorname{Dom}(F) \equiv\{s \in \mathbb{R}$ : $F(s)<\infty\}$. The set $\mathcal{C}(-\Delta, \Omega)$ is a convex cone, that is, it is convex and stable under addition and multiplication by a positive constant.

Example 1. Let $\gamma>\gamma_{d} \equiv d / 2$. Then, as we shall see below,

$$
\sum_{i \in \mathbb{N}^{*}}\left(\lambda_{0, i}\right)^{-\gamma}<\infty
$$

so that the function

$$
F(s)= \begin{cases}s^{-\gamma} & \text { if } s \geq 0 \\ +\infty & \text { if } s<0\end{cases}
$$

belongs to $\mathcal{C}(-\Delta, \Omega)$ and therefore $(-\Delta)^{-\gamma}$ is a trace-class operator.

Example 2. More generally, let $F: \mathbb{R} \rightarrow \mathbb{R} \cup\{+\infty\}$ be a nonincreasing convex function which is non-negative and such that for any $s \geq 0$ large,

$$
F(s) \leq \frac{C}{(1+s)^{\varepsilon+d / 2}},
$$


for some constants $C, \varepsilon>0$. Then we have that

$$
\sum_{i \in \mathbb{N}^{*}} F\left(\lambda_{0, i}\right) \leq \sum_{k \in \mathbb{N}^{*}} F(k) \cdot \# A(k),
$$

where $A(k) \equiv\left\{i \in \mathbb{N}^{*}: k<\lambda_{0, i} \leq k+1\right\}$. Using Weyl's estimate [27], which states that $\# A(k)$ grows like $k^{d / 2-1}$ for large $k$, it follows that $F(k) \cdot \# A(k)$ behaves like $k^{-1-\varepsilon}$ as $k \rightarrow \infty$. Consequently $\sum_{i \in \mathbb{N}^{*}} F\left(\lambda_{0, i}\right)$ is finite and then $F \in \mathcal{C}(-\Delta, \Omega)$.

Example 3. Assume $f: \mathbb{R} \rightarrow \mathbb{R}$ is a Casimir-type function, that is a function that satisfies the following properties:

i) There exists $s_{1} \in[-\infty, \infty)$ such that $f(s)=\infty$ for any $s \in$ $\left(-\infty, s_{1}\right)$.

ii) $f$ is continuous on $\left(s_{1}, \infty\right)$.

iii) There exists $s_{2} \in\left(s_{1}, \infty\right]$ such that $f(s)>0$ for any $s \in\left(s_{1}, s_{2}\right)$ and $f(s)=0$ for any $s \geq s_{2}$.

iv) $f$ is strictly decreasing on $\left(s_{1}, s_{2}\right)$.

v) If $s_{2}=\infty$, there exists two positive constants $\varepsilon$ and $C$ such that for any $s \geq 0$, large,

$$
f(s) \leq \frac{C}{(1+s)^{\varepsilon+1+d / 2}} .
$$

Then the function

$$
F(s)=\int_{s}^{\infty} f(t) d t
$$

falls in the class of functions of Example 2.

Under these conditions $f(-\Delta)$ is also a trace-class operator if one requires $\varepsilon>1$, as shown in [18]. The function of Example 1 above, the Fermi-Dirac statistics defined for $\alpha>0$ by

$$
f(s)=\int_{\mathbb{R}^{d}} \frac{d v}{\alpha+e^{s+|v|^{2} / 2}}
$$

and the Boltzmann distribution

$$
f(s)=e^{-\alpha s}
$$

with $\alpha>0$, are Casimir-type functions.

Consider now the case of a Schrödinger operator $-\Delta+V$, where $V$ is a potential for which there exist eigenvalues $\lambda_{V, 1}<\lambda_{V, 2} \leq \lambda_{V, 3} \leq \ldots$ diverging to infinity and functions such that $\left\{\phi_{V, i}\right\}_{i \in \mathbb{N}^{*}}$ is a complete orthonormal system in $\mathrm{L}^{2}(\Omega)$, where

$$
-\Delta \phi_{V, i}+V \phi_{V, i}=\lambda_{V, i} \phi_{V, i} \quad \text { in } \Omega, \quad \phi_{V, i} \in \mathrm{H}_{0}^{1}(\Omega) .
$$


In this case we define $\mathcal{C}(-\Delta+V, \Omega)$ as the class of functions $F: \mathbb{R} \rightarrow$ $\mathbb{R} \cup\{+\infty\}$ that are convex and such that $\sum_{i \in \mathbb{N}^{*}} F\left(\lambda_{V, i}\right)$ is finite. Then, using spectral theory, we may define for each such $F$ the trace-class operator $F(-\Delta+V)$. We will use these operators extensively in what follows.

2.3. Sobolev-like cones of nuclear operators. We recall that for any $L \in \mathcal{S}_{\infty}$, we denote by $\left\{\left(\nu_{i}(L), \psi_{i}(L)\right)\right\}_{i \in \mathbb{N}^{*}}$ a sequence of eigenelements of $L$. Here $\left\{\psi_{i}(L)\right\} \subset \mathrm{L}^{2}(\Omega)$ is a complete orthonormal system of eigenfunctions.

Definition 2.2. Let $l \in \mathbb{N}^{*}$ and $p \in\left[1, \infty\right.$ [. An operator $L \in \mathcal{S}_{1}$ is in the Sobolev-like cone $\mathcal{W}^{l, p}$ if $\left\{\psi_{i}(L)\right\}_{i \in \mathbb{N}^{*}} \subset \mathrm{W}_{0}^{1, p}(\Omega) \cap \mathrm{W}^{l, p}(\Omega)$ and

$$
\langle\langle L\rangle\rangle_{l, p} \equiv \sum_{i \in \mathbb{N}^{*}}\left|\nu_{i}\right| \cdot\left\|\psi_{i}\right\|_{W^{l, p}(\Omega)}^{p}<\infty .
$$

We also define the functional $\mathcal{K}_{p}$ on $\mathcal{W}^{1, p}$ by

$$
\mathcal{K}_{p}(L) \equiv \sum_{i \in \mathbb{N}^{*}}\left|\nu_{i}\right| \int_{\Omega}\left|\nabla \psi_{i}(x)\right|^{p} d x .
$$

The following proposition collects some basic facts:

Proposition 2.1. Sobolev-like cones of trace-class operators satisfy the following properties:

i) For any $p \in\left[1, \infty\left[, l \in \mathbb{N}^{*}, \mathcal{W}^{l, p}\right.\right.$ is a cone and

$$
\mathcal{W}^{l_{2}, p} \subset \mathcal{W}^{l_{1}, p} \quad \text { if } \quad l_{1} \leq l_{2}, \quad l_{1}, l_{2} \in \mathbb{N}^{*} .
$$

ii) If $1 \leq p<q<\infty, l \in \mathbb{N}^{*}$, then there exists a constant $c_{1}=$ $c_{1}(p, q, l)$ such that

$$
\langle\langle L\rangle\rangle_{l, p} \leq c_{1}\langle\langle L\rangle\rangle_{l, q} \quad \forall L \in \mathcal{W}^{l, q},
$$

so that $\mathcal{W}^{l, q} \subset \mathcal{W}^{l, p}$.

iii) For any $p \geq 2$, there exists a constant $c_{2}=c_{2}(\Omega, p)$ such that

$$
\|L\|_{1} \leq c_{2} \mathcal{K}_{p}(L), \quad \forall L \in \mathcal{W}^{1, p} .
$$

In the rest of the paper we will only consider the case $p=2$, even though some results can be extended for general $p$. In this case we write $\mathcal{H}^{1} \equiv \mathcal{W}^{1,2}$ and we denote by $\mathcal{H}_{+}^{1}$ the set of operators $L \in \mathcal{H}^{1}$ such that $L \geq 0$. This set $\mathcal{H}_{+}^{1}$ is also a cone. We define $\mathcal{K}: \mathcal{H}_{+}^{1} \rightarrow$ $\mathbb{R}$ as $\mathcal{K}=\mathcal{K}_{2}$ and call it the kinetic energy functional. Given $L \in$ $\mathcal{H}_{+}^{1}$ we say that $\mathcal{K}(L)$ is the kinetic energy of $L$ and we may write that $\mathcal{K}(L)=\operatorname{tr}[-\Delta L]$. The Sobolev-like cones $\mathcal{H}^{1}$ and $\mathcal{H}_{+}^{1}$ are the analogues of $\mathrm{H}^{1}(\Omega)$ and $\mathrm{H}_{+}^{1}(\Omega)=\left\{u \in \mathrm{H}^{1}(\Omega): u \geq 0\right\}$ at the level of 
self-adjoint compact operators. This results in integrability properties for the density $\rho_{L}(x)=\sum_{i \in \mathbb{N}^{*}} \nu_{i}\left|\psi_{i}(x)\right|^{2}$ which are the counterpart of Sobolev's embeddings.

Proposition 2.2. For any $L \in \mathcal{H}^{1}$, the density function $\rho_{L}$ belongs to $\mathrm{W}^{1, r}(\Omega) \cap \mathrm{L}^{q}(\Omega)$ with $r$ and $q$ in the following ranges:

i) for all $q \in[1, \infty]$ and $r \in[1,2]$ if $d=1$,

ii) for all $q \in[1, \infty[$ and $r \in[1,2]$ if $d=2$,

iii) for all $q \in[1, d /(d-2)]$ and $r \in[1, d /(d-1)]$ if $d \geq 3$.

Proof. Assume that $d \geq 3$ and $r \in[1, d /(d-1)]$. Using the convexity of $s \mapsto|s|^{r}$, Hölder's and Sobolev's inequalities, we obtain

$$
\begin{aligned}
\int_{\Omega}\left|\nabla \rho_{L}\right|^{r} d x & \leq 2^{r} \int_{\Omega}\left(\sum_{i \in \mathbb{N}^{*}}\left|\nu_{i} \psi_{i} \nabla \psi_{i}\right|\right)^{r} d x \\
& \leq\left(2 \sum_{j \in \mathbb{N}^{*}}\left|\nu_{j}\right|\right)^{r} \int_{\Omega} \sum_{i \in \mathbb{N}^{*}}\left(\frac{\left|\nu_{i}\right|}{\sum_{j \in \mathbb{N}^{*}}\left|\nu_{j}\right|}\right)\left|\psi_{i}\right|^{r}\left|\nabla \psi_{i}\right|^{r} d x \\
& \leq 2^{r}\left(\sum_{j \in \mathbb{N}^{*}}\left|\nu_{j}\right|\right)^{r-1} \sum_{i \in \mathbb{N}^{*}}\left|\nu_{i}\right|\left(\int_{\Omega}\left|\nabla \psi_{i}\right|^{2}\right)^{\frac{r}{2}}\left(\int_{\Omega}\left|\psi_{i}\right|^{\frac{2 r}{2-r}}\right)^{1-\frac{r}{2}} \\
& \leq 2^{r} s_{r}^{r}\|L\|_{1}^{r-1} \mathcal{K}(L)
\end{aligned}
$$

where $s_{r}$ is the Sobolev constant of the embedding $H_{0}^{1}(\Omega) \hookrightarrow L^{\frac{2 r}{2-r}}(\Omega)$. Thus, from (2.7) we find

$$
\left\|\nabla \rho_{L}\right\|_{L^{r}(\Omega)} \leq 2 s_{r}\|L\|_{1}^{1-\frac{1}{r}} \mathcal{K}^{\frac{1}{r}}(L) \leq 2 s_{r} c_{2}^{1-\frac{1}{r}} \mathcal{K}(L)
$$

where $c_{2}=c_{2}(\Omega, 2)$ is the Poincaré constant. Therefore, by the critical Sobolev embedding, we finally have

$$
\left\|\rho_{L}\right\|_{L^{d /(d-2)}(\Omega)} \leq s_{d /(d-1)}\left\|\nabla \rho_{L}\right\|_{L^{d /(d-1)}(\Omega)} \leq 2 s_{d /(d-1)}^{2} c_{2}^{1-\frac{1}{r}} \mathcal{K}(L)<\infty .
$$

The cases $d=1,2$ follow similarly from the Sobolev inequalities, with the corresponding restrictions on $q$ and $r$.

2.4. The free energy functional. In this section we define the free energy functional, which is made of an energy functional and an entropy functional. The energy functional can be seen as the sum of the kinetic energy, as defined above, and a potential energy functional.

2.4.1. Potential energy. Potential energy for trace-class operators can be defined as follows. Let $V: \Omega \rightarrow \mathbb{R}$ be a measurable function and let $L \in \mathcal{S}_{1}$. If $\rho_{|L|} V \in \mathrm{L}^{1}(\Omega)$, then the $V$-potential energy of $L$ is given by

$$
\mathcal{P}_{V}(L) \equiv \int_{\Omega} V(x) \rho_{|L|}(x) d x .
$$


Remark 2.2. Since $V=V(x)$ can formally be seen as an operator acting on $\mathrm{L}^{2}(\Omega)$ with kernel $K_{V}(x, y)=V(x) \delta_{x}(y)$, it follows that $\mathcal{P}(L)=\operatorname{tr}[V|L|]$.

The $V$-potential energy functional is bounded from below in $\mathcal{H}_{+}^{1}$ if and only if $V$ is non-negative. To be precise, we have the following result.

Proposition 2.3. Assume that $A \subseteq \mathcal{S}_{1}$ is such that $\alpha A \subseteq A$, for all $\alpha>0$. Then

$$
\inf _{L \in A} \mathcal{P}(L) \geq C
$$

for some constant $C \in \mathbb{R}$ if and only if

$$
\inf _{L \in A} \mathcal{P}(L)=0
$$

which is equivalent to $V \geq 0$ a.e.

Proof. If we assume (2.8) and there is $L \in A$ such that $0>\mathcal{P}(L)>C$, then it should also be true that

$$
0>\mathcal{P}(\alpha L)=\alpha \mathcal{P}(L)>C \quad \forall \alpha>0,
$$

but this is impossible for $\alpha>|C| /|\mathcal{P}(L)|$. Then, as $\lim _{\alpha \rightarrow 0} \mathcal{P}(\alpha L)=0$, we have (2.9). Next, assuming (2.9) we see that $V \geq 0$ a.e., since in the contrary we can find $L$ such that $\mathcal{P}(L)<0$. Finally, if $V \geq 0$ a.e., then (2.8) follows with $C=0$.

2.4.2. Entropy. Let $L \in \mathcal{S}_{1}$ and let $\beta: \mathbb{R} \rightarrow \mathbb{R} \cup\{+\infty\}$ be a convex function such that $\beta(0)=0$. The functional

$$
\mathcal{E}_{\beta}(L) \equiv \sum_{i \in \mathbb{N}^{*}} \beta\left(\nu_{i}(L)\right)
$$

will be called the $\beta$-entropy of $L$.

At this point the function $\beta$ is arbitrary, but later we consider it in relation with a function $F$ in the class $\mathcal{C}(-\Delta+V, \Omega)$.

2.4.3. Free energy. The free energy functional is obtained as the sum of the entropy, the kinetic energy and the potential energy. Assume that $L \in \mathcal{H}^{1}$ and that $V: \Omega \rightarrow \mathbb{R}$ is a measurable function such that $\rho_{|L|} V \in \mathrm{L}^{1}(\Omega)$.

Definition 2.3. The functional

$$
\mathcal{F}_{V, \beta}(L) \equiv \mathcal{E}_{\beta}(L)+\mathcal{K}(L)+\mathcal{P}_{V}(L)
$$

will be called the $(V, \beta)$-free energy of $L$. 
Formally we may say that the $(V, \beta)$-free energy of $L$ is given by

$$
\mathcal{F}_{V, \beta}(L)=\operatorname{tr}[\beta(L)+(-\Delta+V) L],
$$

for all $L \in \mathcal{H}^{1}$. We recall that we are using the notation $\operatorname{tr}[\cdot]$ when the trace makes sense even though the operator may not be a trace-class operator. See Remark 2.1.

Remark 2.3. Here we take temperature 1 from the physics point of view.

Example 1. Let $\gamma>\gamma_{d} \equiv d / 2$ and

$$
\beta_{m}(s)= \begin{cases}\infty & \text { if } \quad s<0, \\ -c_{m} s^{m} & \text { if } \quad s \geq 0,\end{cases}
$$

where $c_{m}=(1-m)^{m-1} m^{-m}$ and $m=\frac{\gamma}{\gamma+1} \in\left(\frac{d}{d+2}, 1\right)$. The entropy functional $\mathcal{E}_{\beta_{m}}$ associated to $\beta_{m}$ will play an important role in Section 3.1 below. The function $\beta_{m}$ has to be seen in connection with the function $F$ of Example 1 in subsection 2.2.

2.4.4. Poisson potential energy. Let $d \leq 4$. By virtue of Proposition 2.2 we have that $\rho_{L}$ is in $\mathrm{L}^{2}(\Omega)$, for any $L \in \mathcal{H}^{1}$, so that we can find a potential $V_{L} \in \mathrm{H}_{0}^{1}(\Omega)$, called the Poisson potential, as the unique solution of the equation

$$
\left\{\begin{array}{cc}
-\Delta V=\sigma \rho_{L} & \text { in } \quad \Omega, \\
V=0 & \text { on } \quad \partial \Omega .
\end{array}\right.
$$

Two cases can be considered, corresponding either to the repulsive case when $\sigma=+1$ (electrostatic Coulomb interaction), or to the attractive case when $\sigma=-1$ (Newton interaction). The Poisson potential energy of $L \in \mathcal{H}^{1}$ is now defined as

$$
\mathcal{P}(L)=\frac{1}{2} \int_{\Omega} V_{L} \rho_{L} d x=\frac{\sigma}{2} \int_{\Omega}\left|\nabla V_{L}\right|^{2} d x .
$$

Using Proposition 2.2 we get the following regularity result, whose proof can be seen for instance in [10].

Proposition 2.4. Let $L \in \mathcal{H}^{1}$. If $d=1$ or $d=2$, then $V_{L} \in \mathrm{C}^{0}(\bar{\Omega})$. Moreover, $V_{L} \in \mathrm{W}_{0}^{1, p}(\Omega) \cap \mathrm{L}^{q}(\Omega)$ for any $q \in[1, \infty)$ and for any $p \in$ $[1, \infty)$ if $d=3$, and for any $p \in[1,4]$ if $d=4$. If additionally $\partial \Omega$ is of class $\mathrm{C}^{2}$, then $V_{L} \in \mathrm{W}^{2, r}(\Omega) \cap \mathrm{C}^{0,1 / 2}(\Omega)$ for any $r \in[1,3 / 2]$ if $d=3$, and $V_{L} \in \mathrm{W}^{2, r}(\Omega)$ for any $r \in[1,4 / 3]$ if $d=4$.

In Section 4 we will be interested in the more general free energy functional defined as $\mathcal{F}_{V, \beta}(L)+\mathcal{P}(L)$. Such a functional is convex if $\sigma=+1$, but it is not convex if $\sigma=-1$. 


\section{MAin RESUlts}

\subsection{Lieb-Thirring and Gagliardo-Nirenberg inequalities (I).}

In this subsection we interpret the results obtained in [5] in terms of the operator formalism and we adapt those results originally written in $\mathbb{R}^{d}$ to a domain $\Omega \subset \mathbb{R}^{d}$. If $V$ is a potential, with the notation of the end of Subsection 2.2, we let $\left\{\lambda_{V, i}\right\}_{i \in \mathbb{N}^{*}}$ and $\left\{\phi_{V, i}\right\}_{i \in \mathbb{N}^{*}}$ be a sequence of eigenvalues and eigenfunctions of $-\Delta+V$ in $\Omega$, with Dirichlet boundary condition on $\partial \Omega$. We assume that these eigenfunctions define an orthonormal basis for $\mathrm{L}^{2}(\Omega)$.

Following the setting defined in [5], we let $g$ be a non-negative function on $\mathbb{R}^{+}$such that

$$
\int_{0}^{\infty} g(t)\left(1+t^{-d / 2}\right) \frac{d t}{t}<\infty
$$

and we define

$$
F(s)=\int_{0}^{\infty} e^{-t s} g(t) \frac{d t}{t} \text { and } \quad G(s)=\int_{0}^{\infty} e^{-t s}(4 \pi t)^{-d / 2} g(t) \frac{d t}{t} .
$$

We observe that $F, G: \mathbb{R} \rightarrow \mathbb{R} \cup\{+\infty\}$ are convex non-increasing functions.

If $V \in L_{\text {loc }}^{1}\left(\mathbb{R}^{d}\right)$ is bounded from below and $G(V) \in L^{1}\left(\mathbb{R}^{d}\right)$, with $F$ and $G$ given as in (3.2) and $g$ satisfying (3.1), then the content of Theorem 3 in [5] is the following Lieb-Thirring inequality

$$
\operatorname{Tr}[F(-\Delta+V)] \leq \int_{\mathbb{R}^{d}} G(V(x)) d x .
$$

We can extend this result so to consider a domain $\Omega$ instead of $\mathbb{R}^{d}$. Precisely we have

Theorem 3.1. Let $\Omega$ be a domain and $V$ be a potential bounded from below and in $L_{\text {loc }}^{1}(\Omega)$. Assume moreover that $G(V)$ is in $L^{1}(\Omega)$, with $F$ and $G$ given by (3.2) and $g$ satisfying (3.1). Then we have

$$
\sum_{i \in \mathbb{N}^{*}} F\left(\lambda_{V, i}\right)=\operatorname{Tr}[F(-\Delta+V)] \leq \int_{\Omega} G(V(x)) d x .
$$

A proof of this theorem is easily achieved using Theorem 3 in [5] with an appropriate increasing sequence of potentials $\left\{V_{n}\right\}$, so that its limit is $+\infty$ outside $\Omega$ and $V$ in $\Omega$.

We notice that since $V$ is bounded below, letting $\lambda<\operatorname{infess}_{\Omega} V$, we see that the eigenvalues $\lambda_{V, i}$ satisfy $\lambda_{V, i} \geq \lambda_{0, i}+\lambda$, for all $i \in \mathbb{N}^{*}$, and then the sequence $\left\{\lambda_{V, i}\right\}_{i \in \mathbb{N}^{*}}$ diverges, since the sequence $\left\{\lambda_{0, i}\right\}_{i \in \mathbb{N}^{*}}$ diverges. Under the hypotheses of Theorem 3.1, the function $F$ given 
by (3.2), is convex and satisfies $\sum_{i \in \mathbb{N}^{*}} F\left(\lambda_{V, i}\right)<\infty$ so that $F$ belongs to the class $\mathcal{C}(-\Delta+V, \Omega)$. Theorem 3.1 can be illustrated by the following examples.

Example 1. If $F(s)=s^{-\gamma}$ for any $s \geq 0$ and $F(s)=+\infty$ for $s<0$, then $G(s)=\mathcal{C}(\gamma) s^{\frac{d}{2}-\gamma}$ for $s \geq 0$, where $\mathcal{C}(\gamma)=(4 \pi)^{-d / 2} \Gamma\left(\gamma-\frac{d}{2}\right) / \Gamma(\gamma)$. In such a case, Theorem 3.1 takes the following special form

Corollary 3.1. Let $\Omega$ be a domain in $\mathbb{R}^{d}$ and $V \in L_{\text {loc }}^{1}(\Omega)$ a potential bounded below. Assume moreover that $(V)^{\frac{d}{2}-\gamma}$ is in $L^{1}(\Omega)$ for some $\gamma>d / 2$. Then

$$
\operatorname{Tr}\left[(-\Delta+V)^{-\gamma}\right]=\sum_{i \in \mathbb{N}^{*}}\left(\lambda_{V, i}\right)^{-\gamma} \leq \frac{\Gamma\left(\gamma-\frac{d}{2}\right)}{(4 \pi)^{d / 2} \Gamma(\gamma)} \int_{\Omega} V^{\frac{d}{2}-\gamma} d x .
$$

Example 2. If $F(s)=e^{-s}$ for any $s \in \mathbb{R}$, then $G(s)=(4 \pi)^{-d / 2} e^{-s}$ and Theorem 3.1 reads as follows

Corollary 3.2. Let $\Omega$ be a domain in $\mathbb{R}^{d}$ and $V$ a potential in $L_{\text {loc }}^{1}(\Omega)$. If $e^{-V} \in L^{1}(\Omega)$, then we have

$$
\operatorname{Tr}\left[e^{-(-\Delta+V)}\right]=\sum_{i \in \mathbb{N}^{*}} e^{-\lambda_{V, i}} \leq \frac{1}{(4 \pi)^{d / 2}} \int_{\Omega} e^{-V} d x .
$$

Proof. To prove this result, assume first that $V$ is bounded from below and apply Theorem 3.1. One concludes for general potentials $V$ by density.

In Section 3.3 we will see how to extend the results of Theorem 3.1 to general potentials that may be unbounded from below, but that still have some boundedness property with respect to $-\Delta$.

Now we are going to state some Gagliardo-Nirenberg inequalities in the context of the operator formalism. In the way to get them, we will obtain some useful estimates for $\mathcal{F}_{V, \beta}$.

Given a convex function $\theta: \mathbb{R} \rightarrow \mathbb{R} \cup\{+\infty\}$ such that $\theta \not \equiv+\infty$, we shall denote by $\theta^{*}$ the Legendre-Fenchel transform of $\theta$, that is the function defined by

$$
\theta^{*}(\nu) \equiv \sup _{\lambda \in \mathbb{R}}\{\nu \lambda-\theta(\lambda)\} \quad \forall \nu \in \mathbb{R}
$$

Thus, if $F$ is convex and $\beta$ is given by $\beta(s)=F^{*}(-s)$ for all $s \in \mathbb{R}$, we get

$$
\beta(\nu)+\nu \lambda \geq-F(\lambda) \quad \forall \nu, \lambda \in \mathbb{R} .
$$


From here we get a uniform lower bound for $\mathcal{F}_{V, \beta}$ on $\mathcal{H}_{+}^{1}$ under the conditions of Theorem 3.1. That is, in terms of operators, we have

$$
\mathcal{F}_{V, \beta}(L) \geq-\operatorname{Tr}[F(-\Delta+V)] \quad \forall L \in \mathcal{H}_{+}^{1} .
$$

In order to prove this, let $\psi \in H_{0}^{1}(\Omega)$ such that $\|\psi\|_{L^{2}(\Omega)}=1$. Then there exists a sequence $\left\{\alpha_{i}\right\}_{i \in \mathbb{N}^{*}} \subset \mathbb{R}$ such that $\psi=\sum_{i \in \mathbb{N}^{*}} \alpha_{i} \phi_{V, i}$ and $\sum_{i \in \mathbb{N}^{*}} \alpha_{i}^{2}=1$. By convexity of $F$, we obtain

$$
\begin{aligned}
F\left(\int_{\Omega}|\nabla \psi|^{2} d x\right. & \left.+\int_{\Omega} V|\psi|^{2} d x\right)=F\left(\sum_{i \in \mathbb{N}^{*}} \alpha_{i}^{2} \lambda_{0, i}\right) \\
\leq \sum_{i \in \mathbb{N}^{*}} \alpha_{i}^{2} F\left(\lambda_{0, i}\right) & =\langle\psi, F(-\Delta+V) \psi\rangle_{\mathrm{L}^{2}(\Omega)} .
\end{aligned}
$$

If $\psi$ is an eigenfunction of $-\Delta+V$ then this inequality becomes an equality. Using (3.3), we can now bound from below the free energy. Substituting $\nu_{i}$ for $\nu, \int_{\Omega}\left(\left|\nabla \psi_{i}\right|^{2}+V\left|\psi_{i}\right|^{2}\right) d x$ for $\lambda$ and adding over $i \in \mathbb{N}^{*}$, we get

$$
\begin{aligned}
\mathcal{E}_{\beta}(L)+\mathcal{K}(L) & +\mathcal{P}(L)=\sum_{i \in \mathbb{N}^{*}}\left[\beta\left(\nu_{i}\right)+\nu_{i} \int_{\Omega}\left(\left|\nabla \psi_{i}\right|^{2}+V\left|\psi_{i}\right|^{2}\right) d x\right] \\
& \geq-\sum_{i \in \mathbb{N}^{*}} F\left(\int_{\Omega}\left|\nabla \psi_{i}\right|^{2} d x+\int_{\Omega} V\left|\psi_{i}\right|^{2} d x\right) \\
& \geq-\sum_{i \in \mathbb{N}^{*}}\left\langle\psi_{i}, F(-\Delta+V) \psi_{i}\right\rangle_{\mathrm{L}^{2}(\Omega)}=-\operatorname{Tr}[F(-\Delta+V)] .
\end{aligned}
$$

Hence we obtain (3.4).

Now, if $F$ and $G$ are as in Theorem 3.1, then we have

$$
-\operatorname{Tr}[F(-\Delta+V)] \geq-\int_{\Omega} G(V(x)) d x
$$

and

$$
\mathcal{E}_{\beta}(L)+\mathcal{K}(L)+\mathcal{J}_{L}(V)=\mathcal{F}_{V, \beta}(L)+\int_{\Omega} G(V(x)) d x \geq 0,
$$

for all $L \in \mathcal{H}_{+}^{1}$, where we define

$$
\mathcal{J}_{L}(V) \equiv \int_{\Omega}\left[V(x) \rho_{L}(x)+G(V(x))\right] d x
$$

Next we proceed as in [5] minimizing $\mathcal{J}_{L}(\cdot)$ in the set of potentials $V$ which verify $(3.4)$ and $(3.5)$, for $L$ fixed. If $V_{0}$ is the minimizer of $\mathcal{J}_{L}(\cdot)$, then

$$
G^{\prime}\left(V_{0}\right)+\rho_{L}=0
$$


By defining $\tau(s) \equiv-\left[\left(G \circ\left(G^{\prime}\right)^{-1}\right)(-s)+s\left(G^{\prime}\right)^{-1}(-s)\right]$, that is $\tau$ such that

$$
G(s)=\tau^{*}(-s)
$$

we have that

$$
\mathcal{J}_{L}\left(V_{0}\right)=-\int_{\Omega} \tau\left(\rho_{L}(x)\right) d x
$$

and we can state the following

Theorem 3.2. Let $\Omega$ be a domain and functions $F$ and $G$ be defined as in (3.2). Let us consider $\beta$ and $\tau$ such that $\beta(s) \equiv F^{*}(-s)$ and $G(s) \equiv \tau^{*}(-s)$. Then for any $L \in \mathcal{H}_{+}^{1}$, we have

$$
\mathcal{K}(L)+\mathcal{E}_{\beta}(L) \geq \int_{\Omega} \tau\left(\rho_{L}\right) d x .
$$

Since $G$ is convex, (3.6) holds even if $G$ is not differentiable. This theorem provides interesting insights in the following two typical examples.

Example 1. Assume that $\gamma>\gamma_{d} \equiv d / 2$ and consider the convex function $\beta_{m}(s)=-c_{m} s^{m}$ on $\mathbb{R}^{+}$, extended by $+\infty$ on $(-\infty, 0)$, where $c_{m}=$ $(1-m)^{m-1} m^{-m}, m=\frac{\gamma}{\gamma+1} \in\left(\frac{d}{d+2}, 1\right)$. The corresponding functions $F$ and $G$ take the form $F(s)=\beta_{m}^{*}(-s)=s^{-\gamma}$ and $G(s)=\mathcal{C}(\gamma) s^{\frac{d}{2}-\gamma}$ for $s \geq 0$, extended by $+\infty$ to the interval $(-\infty, 0)$.

Define

$$
q=\frac{2 \gamma-d}{2(\gamma+1)-d} \in(0,1)
$$

In such a case, Theorem 3.2 takes the following special form

Corollary 3.3. With the above notations, for any $L \in \mathcal{H}_{+}^{1}$,

$$
\mathcal{K}(L)+\kappa(\gamma) \int_{\Omega} \rho_{L}^{q} d x \geq c_{m} \operatorname{Tr}\left[L^{m}\right]
$$

where $\kappa(\gamma) \equiv(\mathcal{C}(\gamma))^{1-q}\left[\left(\frac{q}{q-1}\right)^{1-q}+\left(\frac{q}{q-1}\right)^{-q}\right]$.

Example 2. Consider the convex function $\beta_{1}(s) \equiv s \log s-s$ if $s>0$, extended by $\beta_{1}(0)=0$ and $+\infty$ on $(-\infty, 0)$, and corresponding functions $F(s)=e^{-s}$ and $G(s)=(4 \pi)^{-d / 2} e^{-s}$, for any $s \in \mathbb{R}$. Theorem 3.2 applies as follows

Corollary 3.4. For any $L \in \mathcal{H}_{+}^{1}$,

$$
\mathcal{K}(L)+\operatorname{Tr}[L \log L] \geq \int_{\Omega} \rho_{L} \log \rho_{L} d x+\frac{d}{2} \log (4 \pi) \int_{\Omega} \rho_{L} d x .
$$


3.2. Convexity Estimates. In this subsection we extend some of the ideas discussed before to include potential functions which are not necessarily bounded from below. In [18, Lemma 3], in the context of mixed states, the authors obtained an estimate like (3.4), for functions $F$ generated by a Casimir-type functions $f$, in presence of a repulsive Poisson coupling and for $d=3$. See Example 3 in Section 2.2 above. Here we want to establish such a type of estimate for potential which may be not only negative, but also not bounded from below.

We first establish a direct consequence of (3.4), for non-negative potentials. With obvious notations, we say that $F \in \mathcal{C}(-(1-\varepsilon) \Delta+V, \Omega)$ if $F$ is convex and $\sum_{i \in \mathbb{N}^{*}} F\left(\lambda_{V, i}^{\varepsilon}\right)$ is finite, where $\lambda_{V, i}^{\varepsilon}$ are the eigenvalues of $-(1-\varepsilon) \Delta+V$ in $\Omega$, with Dirichlet boundary condition.

Lemma 3.1. Assume that $V$ is a non-negative potential, $\varepsilon \in(0,1]$ and $F \in \mathcal{C}(-\varepsilon \Delta+V, \Omega)$. If $\beta(s) \equiv F^{*}(-s)$ then for any $L \in \mathcal{H}_{+}^{1}$ we have

$$
\mathcal{E}_{\beta}(L)+(1-\varepsilon) \mathcal{K}(L)+\mathcal{P}_{V}(L) \geq-\operatorname{Tr}[F(-(1-\varepsilon) \Delta+V)]
$$

and

$$
\mathcal{F}_{V, \beta}(L) \geq \varepsilon \mathcal{K}(L)-\operatorname{Tr}[F(-(1-\varepsilon) \Delta+V)] .
$$

In order to extend our approach to potentials which take negative values, some additional definitions are needed.

Definition 3.1. We will say that the Schrödinger operator $-\Delta+V$ is $\varepsilon$ coercive for some $\varepsilon \in(0,1]$ if and only if

$$
\lambda_{V, 1}^{\varepsilon} \equiv \sup \{\mu \in \mathbb{R}:-(1-\varepsilon) \Delta+V \geq \mu\}>-\infty .
$$

The $\varepsilon$ coercivity indeed means that

$$
-\Delta+V-\lambda_{V, 1}^{\varepsilon} \geq-\varepsilon \Delta
$$

in the sense of operators. We will denote by $\left\{\lambda_{V, i}^{\varepsilon}\right\}_{i \in \mathbb{N}^{*}}$ the sequence of eigenvalues of the operator $-(1-\varepsilon) \Delta+V$. Notice that $\lambda_{V, 1}^{0}=\lambda_{V, 1}$. We also observe that condition (3.7) for $\varepsilon=1$ means that $V$ is bounded from below and $\lambda_{V, 1}^{1}=0$ means $V$ is non-negative.

For $\lambda \leq \lambda_{V, i}^{\varepsilon}$ we define the free energy functional $\mathcal{F}_{V, \beta}^{\lambda}: \mathcal{H}_{+}^{1} \rightarrow$ $\mathbb{R} \cup\{\infty\}$ as

$$
\mathcal{F}_{V, \beta}^{\lambda}(L) \equiv \mathcal{F}_{V, \beta}(L)-\lambda \operatorname{Tr}[L] .
$$

In order to obtain a lower bound for this functional, we assume that the function $F \in \mathcal{C}(-(1-\varepsilon) \Delta+V+\lambda, \Omega)$, that is $F: \mathbb{R} \rightarrow \mathbb{R} \cup\{+\infty\}$ is convex and $\sum_{i \in \mathbb{N}^{*}} F\left(\lambda_{V, i}^{\varepsilon}-\lambda\right)$ is finite.

We notice that if $F \in \mathcal{C}(-(1-\varepsilon) \Delta+V+\lambda, \Omega)$, then $\left[\lambda_{V, 1}^{\varepsilon}-\lambda, \infty\right) \subset$ $\operatorname{Dom}(F)$ even if $V-\lambda$ may be negative at some open sets. With the same convexity argument as in the proof of (3.4) and Lemma 3.1, we 
can see that if $V$ verifies condition (3.7) for a given $\varepsilon \in[0,1]$, then for any $F \in \mathcal{C}(-(1-\varepsilon) \Delta+V+\lambda, \Omega)$, with $\beta(s)=F^{*}(-s), s \in \mathbb{R}$

$$
\mathcal{F}_{V, \beta}^{\lambda}(L) \geq \varepsilon \mathcal{K}(L)-\operatorname{Tr}[F(-(1-\varepsilon) \Delta+V-\lambda)] \quad \forall L \in \mathcal{H}_{+}^{1},
$$

so that $\mathcal{F}_{V, \beta}^{\lambda}(L)$ is uniformly bounded from below. Moreover we see that $\|L\|_{1}$ is uniformly bounded, thanks to (2.7), and in this sense $\mathcal{F}_{V, \beta}^{\lambda}$ is coercive.

We formalize these conclusions in the following proposition

Proposition 3.5. Let $V$ be a potential verifying (3.7) for some $\varepsilon \in$ $(0,1]$. For any $F \in \mathcal{C}\left(-\frac{\varepsilon}{2} \Delta, \Omega\right)$, let $\beta(s) \equiv F^{*}(-s), s \in \mathbb{R}$. For any $\lambda \leq \lambda_{V, 1}^{\varepsilon}$, for any $L \in \mathcal{H}_{+}^{1}$,

$$
\mathcal{F}_{V, \beta}^{\lambda}(L) \geq-\operatorname{Tr}\left[F\left(-\frac{\varepsilon}{2} \Delta\right)\right]+\frac{\varepsilon}{2} \mathcal{K}(L) .
$$

Hence $\mathcal{F}_{V, \beta}^{\lambda}$ is bounded from below and

$$
\mathcal{F}_{V, \beta}^{\lambda}(L) \geq \operatorname{Tr}[F(-\Delta+V-\lambda)] \quad \forall L \in \mathcal{H}_{+}^{1} .
$$

Moreover, if $\mathcal{F}_{V, \beta}^{\lambda}(L)$ is finite for some $L \in \mathcal{H}_{+}^{1}$, then $-\Delta+V$ has only pure point spectrum, provided $F$ is positive on $(0,+\infty)$.

Proof. Let $L \in \mathcal{H}^{1}$ and write

$\mathcal{F}_{V, \beta}^{\lambda}(L)=\left\{\mathcal{E}_{\beta}(L)+\frac{\varepsilon}{2} \mathcal{K}(L)\right\}+\frac{\varepsilon}{2} \mathcal{K}(L)+\left\{(1-\varepsilon) \mathcal{K}(L)+\mathcal{P}_{V}(L)-\lambda\|L\|_{1}\right\}$.

From Lemma 3.1 we have that

$$
\mathcal{E}_{\beta}(L)+\frac{\varepsilon}{2} \mathcal{K}(L) \geq-\operatorname{Tr}\left[F\left(-\frac{\varepsilon}{2} \Delta\right)\right] .
$$

On the other hand, from (3.7) it follows that

$$
\begin{aligned}
(1-\varepsilon) & \mathcal{K}(L)+\mathcal{P}_{V}(L)-\lambda\|L\|_{1} \\
= & \sum_{i \in \mathbb{N}^{*}} \nu_{i} \int_{\Omega}\left((1-\varepsilon)\left|\nabla \psi_{i}\right|^{2}+(V-\lambda)\left|\psi_{i}\right|^{2}\right) d x \geq 0,
\end{aligned}
$$

where $\left(\nu_{i}, \psi_{i}\right)=\left(\nu_{i}(L), \psi_{i}(L)\right)$ are a complete sequence of eigenpairs of the operator $L$, with the notation of Section 2.1. This proves the lower bound on $\mathcal{F}_{V, \beta}^{\lambda}(L)$.

The assertion on the spectrum of $-\Delta+V$ easily follows. Notice that from its definition, $F$ is non-negative. Since $\sum_{i \in \mathbb{N}^{*}} F\left(\lambda_{V, i}-\lambda\right)<\infty$, to prove that $\left\{\lambda_{V, i}\right\}_{i \in \mathbb{N}^{*}}$ diverges, it is therefore sufficient to require that $F$ is positive.

Corollary 3.6. Under the conditions of Proposition 3.5, if $\left\{L_{n}\right\}_{n \in \mathbb{N}^{*}}$ is a sequence in $\mathcal{H}_{+}^{1}$ such that $\left\{\mathcal{F}_{V, \beta}^{\lambda}\left(L_{n}\right)\right\}_{n \in \mathbb{N}^{*}}$ is bounded, then the sequences $\left\{\left\|L_{n}\right\|_{1}\right\}_{n \in \mathbb{N}^{*}},\left\{\mathcal{K}\left(L_{n}\right)\right\}_{n \in \mathbb{N}^{*}},\left\{\mathcal{E}_{\beta}\left(L_{n}\right)\right\}_{n \in \mathbb{N}^{*}}$ and $\left\{\mathcal{P}_{V}\left(L_{n}\right)\right\}_{n \in \mathbb{N}^{*}}$ are also bounded. 
Proof. As follows from (3.8) and (3.9) in the proof of Proposition 3.5, it is clear that the boundedness of $\mathcal{F}_{V, \beta}^{\lambda}\left(L_{n}\right)$ implies the boundedness from above of $(1-\varepsilon) \mathcal{K}\left(L_{n}\right)+\mathcal{P}_{V}\left(L_{n}\right)-\lambda\left\|L_{n}\right\|_{1}$ and of $\mathcal{E}_{\beta}$. Then we obtain the boundedness from above of $\mathcal{K}\left(L_{n}\right)$ and therefore that of $\left\|L_{n}\right\|_{1}$, by (2.7). Now the boundedness of $\mathcal{P}_{V}\left(L_{n}\right)$ follows.

3.3. Lieb-Thirring and Gagliardo-Nirenberg inequalities (II). In Section 3.1 we established Lieb-Thirring inequalities only for potentials bounded from below. Under condition (3.7) for some $\varepsilon \in(0,1)$, a Lieb-Thirring inequality also holds and then an interpolation inequality can also be established for such potentials. This will give a proof of Theorem 1.1.

To start with, we use scaling to rewrite Theorem 3.1 with $-\Delta$ replaced by $-\varepsilon \Delta$.

Lemma 3.2. Let $\varepsilon \in(0,1)$ and consider a non-negative potential $W$. Let $F$ and $G$ be defined by (3.2), with $g$ satisfying (3.1). Consider $\beta$ and $\tau$ such that $\beta(s) \equiv F^{*}(-s)$ and $G(s) \equiv \tau^{*}(-s)$, with the notations of Section 3.1. Then, for any $L \in \mathcal{H}_{+}^{1}$,

$\operatorname{tr}[\beta(L)+(-\varepsilon \Delta+W) L] \geq-\operatorname{Tr}[F(-\varepsilon \Delta+W)] \geq-\varepsilon^{-\frac{d}{2}} \int_{\Omega} G(W) d x$.

Proof. Let $\left\{\psi_{i}\right\}=\left\{\psi_{i}(L)\right\}$ be a complete basis of $L^{2}(\Omega)$ of eigenvectors of $L$. Then we consider the scaling $\psi_{i}^{\varepsilon}(x)=\varepsilon^{d / 4} \psi_{i}(\sqrt{\varepsilon} x), W^{\varepsilon}(x)=$ $W(\sqrt{\varepsilon} x)$ for any $x \in \varepsilon^{-1 / 2} \Omega$ and denote by $L^{\varepsilon}$ the operator associated to $L$ after the scaling. Then

$$
\int_{\Omega}\left(\varepsilon\left|\nabla \psi_{i}\right|^{2}+W\left|\psi_{i}\right|^{2}\right) d x=\int_{\varepsilon^{-1 / 2} \Omega}\left(\left|\nabla \psi_{i}^{\varepsilon}\right|^{2}+W^{\varepsilon}\left|\psi_{i}^{\varepsilon}\right|^{2}\right) d x
$$

for all $i \in \mathbb{N}^{*}$ and then

$$
\operatorname{tr}[\beta(L)+(-\varepsilon \Delta+W) L]=\operatorname{tr}\left[\beta\left(L^{\varepsilon}\right)+\left(-\Delta+W^{\varepsilon}\right) L^{\varepsilon}\right] .
$$

Applying the results of Theorem 3.1 we find

$$
\operatorname{tr}\left[\beta\left(L^{\varepsilon}\right)+\left(-\varepsilon \Delta+W^{\varepsilon}\right) L^{\varepsilon}\right] \geq-\operatorname{Tr}\left[F\left(-\Delta+W^{\varepsilon}\right)\right] \geq-\int_{\varepsilon^{-1 / 2} \Omega} G\left(W^{\varepsilon}\right) d x .
$$

The conclusion then holds by undoing the change of variables.

Now we consider $\lambda \leq \lambda_{V, 1}^{\varepsilon}$. For any $L \in \mathcal{H}_{+}^{1}$, using the definition of $\mathcal{F}_{V+W, \beta}^{\lambda}$, of $\lambda_{V, 1}^{\varepsilon}$ and Lemma 3.2 we find that

$$
\begin{aligned}
\mathcal{F}_{V+W, \beta}^{\lambda}(L) & =\operatorname{tr}[(-(1-\varepsilon) \Delta+V-\lambda) L]+\operatorname{tr}[\beta(L)+(-\varepsilon \Delta+W) L] \\
& \geq-\varepsilon^{-\frac{d}{2}} \int_{\Omega} G(W) d x .
\end{aligned}
$$


Then we can rearrange this estimate as

$$
\mathcal{F}_{V, \beta}^{\lambda}(L) \geq-\int_{\Omega}\left(\rho_{L} W+\varepsilon^{-\frac{d}{2}} G(W)\right) d x .
$$

Optimizing on $W$ as in Section 3.1, we get that the right hand side is bounded from below by

$$
\varepsilon^{-\frac{d}{2}} \int_{\Omega} \tau\left(\varepsilon^{\frac{d}{2}} \rho_{L}(x)\right) d x
$$

which completes the proof of Theorem 1.1.

3.4. Compactness results. Let us start with some observations. Assume that $\left\{L_{n}\right\}_{n \in \mathbb{N}}$ is a bounded sequence in $\mathcal{S}_{1}$ and denote respectively by $\left\{\nu_{i}^{n}\right\}_{i \in \mathbb{N}^{*}}$ and $\left\{\psi_{i}^{n}\right\}_{i \in \mathbb{N}^{*}}$ the sequence of eigenvalues and a sequence of orthonormalized eigenfunctions of $L_{n}$. Then there exists a constant $C>0$ such that $\left|\nu_{i}^{n}\right| \leq C$, for all $i, n \in \mathbb{N}^{*}$ and, consequently, there exists a sequence of real numbers $\left\{\bar{\nu}_{i}\right\}_{i \in \mathbb{N}^{*}}$ such that, up to a subsequence,

$$
\lim _{n \rightarrow \infty} \nu_{i}^{n}=\bar{\nu}_{i}, \quad \forall i \in \mathbb{N}^{*} .
$$

Our first result is concerned with the case of Example 1 in Section 3.1. We easily see that Theorem 1.2 is a direct consequence of this result and Corollary 3.6.

Theorem 3.3. Consider a bounded domain $\Omega$ in $\mathbb{R}^{d}, d \geq 2$, and assume that $m \in\left(\frac{d}{d+2}, 1\right)$. Let $\left\{L_{n}\right\}_{n \in \mathbb{N}}$ be a sequence in $\mathcal{H}^{1}$ such that

$$
K_{\infty} \equiv \sup _{n \in \mathbb{N}} \mathcal{K}\left(L_{n}\right)<\infty,
$$

for some constant $K_{\infty}>0$. Then, $\left\{L_{n}\right\}_{n \in \mathbb{N}}$ is bounded in $\mathcal{S}_{1}$ and

$$
\sup _{n \in \mathbb{N}} \sum_{i \in \mathbb{N}^{*}}\left|\nu_{i}^{n}\right|^{m}<\infty
$$

Moreover, the following properties hold:

i) If $\bar{\nu}_{i} \neq 0$ for all $i \in \mathbb{N}^{*}$, then, up to a subsequence,

$$
\lim _{n \rightarrow \infty} \sum_{i \in \mathbb{N}^{*}}\left|\nu_{i}^{n}\right|^{m}=\sum_{i \in \mathbb{N}^{*}}\left|\bar{\nu}_{i}\right|^{m} .
$$

ii) For any $m^{\prime} \in(m, 1]$, up to a subsequence,

$$
\lim _{n \rightarrow \infty} \sum_{i \in \mathbb{N}^{*}}\left|\nu_{i}^{n}\right|^{m^{\prime}}=\sum_{i \in \mathbb{N}^{*}}\left|\bar{\nu}_{i}\right|^{m^{\prime}}
$$

iii) Up to a subsequence, $\left\{L_{n}\right\}_{n \in \mathbb{N}}$ converges to some $L$ in $\mathcal{S}_{1}$ and $\left\{K_{L_{n}}\right\}_{n \in \mathbb{N}}$ converges $K_{L}$ in $L^{2}(\Omega \times \Omega)$. 
Proof. By (2.7), $\sup _{n \in \mathbb{N}}\left\|L_{n}\right\|_{1}<\infty$. For $i \in \mathbb{N}^{*}, n \in \mathbb{N}$, let

$$
E_{i}^{n} \equiv \int_{\Omega}\left|\nabla \psi_{i}^{n}(x)\right|^{2} d x
$$

The uniform bound on $\left\|L_{n}\right\|_{1}$ and $\sum_{i \in \mathbb{N}^{*}}\left|\nu_{i}^{n}\right|^{m}$ follow from Proposition 2.2, and Corollary 3.3 and Hölder's inequality respectively.

Proof of i) Assume first that $\bar{\nu}_{i} \neq 0$ for any $i \in \mathbb{N}^{*}$. Then, for each $i \in \mathbb{N}^{*}$, the sequence $\left\{E_{i}^{n}\right\}_{n \in \mathbb{N}}$ is bounded and, consequently, there is a function $\bar{\psi}_{i} \in \mathrm{L}^{2}(\Omega)$ for which, up to a subsequence,

$$
\lim _{n \rightarrow \infty} \psi_{i}^{n}=\bar{\psi}_{i} \quad \text { in } \mathrm{L}^{2}(\Omega) .
$$

Recall that, counting multiplicity, $\left|\bar{\nu}_{1}\right| \geq\left|\bar{\nu}_{2}\right| \geq \ldots$ We denote by $P_{N}$ : $L^{2}(\Omega) \rightarrow F_{N}$ the orthogonal projection operator over

$$
F_{N} \equiv \operatorname{span}\left\{\bar{\psi}_{i}: 1 \leq i \leq N-1\right\}
$$

and let $Q_{N} \equiv I_{d}-P_{N}$ be the projection operator onto $F_{N}^{\perp}$.

Next we claim that for all $\varepsilon>0$, there exists $N \in \mathbb{N}^{*}$ such that

$$
\sum_{i=N}^{\infty}\left|\nu_{i}^{n}\right|^{m} \leq \varepsilon \quad \forall n \in \mathbb{N}
$$

This can be proved as follows. First, using (2.4), we choose $N \in \mathbb{N}^{*}$ such that

$$
\left(\sum_{\ell=N}^{\infty}\left(\lambda_{0, \ell}\right)^{-\gamma}\right)^{m / \gamma} \leq \frac{\varepsilon}{2}
$$

where $\gamma=\frac{m}{1-m}$ and $\left\{\lambda_{0, i}\right\}_{i \in \mathbb{N}^{*}}$ is the sequence of the eigenvalues of $-\Delta$ in $\mathrm{H}_{0}^{1}(\Omega)$, with associated eigenfunctions $\phi_{0, i}, i \in \mathbb{N}^{*}$. Consider for each $n \in \mathbb{N}$ the expansion

$$
\psi_{i}^{n}=\sum_{k=1}^{\infty} \alpha_{i, k}^{n} \phi_{0, k} \quad n \in \mathbb{N},
$$

where $\alpha_{i, k}^{n} \equiv\left\langle\psi_{i}^{n}, \phi_{0, k}\right\rangle_{\mathrm{L}^{2}(\Omega)}$. According to the reverse Hölder inequality, which states that for any $p \in(0,1), q \in(-\infty, 0)$ such that $\frac{1}{p}+\frac{1}{q}=1$,

$$
\sum_{i \in \mathbb{N}^{*}} a_{i} b_{i} \geq\left(\sum_{i \in \mathbb{N}^{*}} a_{i}^{p}\right)^{1 / p}\left(\sum_{i \in \mathbb{N}^{*}} b_{i}^{q}\right)^{1 / q} \forall\left\{a_{i}\right\}_{i \in \mathbb{N}^{*}},\left\{b_{i}\right\}_{i \in \mathbb{N}^{*}} \in\left(\mathbb{R}^{+}\right)^{\mathbb{N}^{*}}
$$


applied for $p=m=\gamma /(\gamma+1), q=-\gamma, a_{i}=\left|\nu_{i}^{n}\right|$ and $b_{i}=E_{i}^{n}$, we get, for all $N \in \mathbb{N}^{*}$, that

$$
\left(\sum_{i=N}^{\infty}\left|\nu_{i}^{n}\right|^{m}\right)^{1 / m} \leq K_{\infty}\left(\sum_{i=N}^{\infty}\left(E_{i}^{n}\right)^{-\gamma}\right)^{1 / \gamma} .
$$

Next we find $N \in \mathbb{N}^{*}$ large enough so that

$$
\left\|P_{N}\left(\phi_{0, \ell}\right)\right\|_{L^{2}(\Omega)} \geq 1-\frac{1}{2} \varepsilon^{\gamma / m} \quad \ell=1,2, \ldots N-1,
$$

or, which is equivalent,

$$
\left\|Q_{N}\left(\phi_{0, \ell}\right)\right\|_{L^{2}(\Omega)} \leq \frac{1}{2} \varepsilon^{\gamma / m}, \quad \ell=1,2, \ldots N-1 .
$$

Then, there is $n_{0} \in \mathbb{N}^{*}$ large enough so that,

$$
\sum_{i=N}^{\infty}\left(\alpha_{i, \ell}^{n}\right)^{2} \leq \varepsilon^{\gamma / m} \quad \forall n \geq n_{0}, \ell=1,2, \ldots, N-1 .
$$

Using $E_{i}^{n}=\sum_{\ell=1}^{\infty} \lambda_{0, \ell}\left(\alpha_{i, \ell}^{n}\right)^{2}$ and $\sum_{\ell=1}^{\infty}\left(\alpha_{i, \ell}^{n}\right)^{2}=1$, by concavity of $s \mapsto s^{-\gamma}$ we have

$$
\left(E_{i}^{n}\right)^{-\gamma} \leq \sum_{\ell=1}^{\infty}\left(\alpha_{i, \ell}^{n}\right)^{2}\left(\lambda_{0, \ell}\right)^{-\gamma}
$$

Hence, collecting the above estimates, we obtain

$$
\begin{aligned}
\sum_{i=N}^{\infty}\left(E_{i}^{n}\right)^{-\gamma} \leq \sum_{i=N}^{\infty} \sum_{\ell=1}^{\infty}\left(\alpha_{i, \ell}^{n}\right)^{2}\left(\lambda_{0, \ell}\right)^{-\gamma} & =\sum_{\ell=1}^{M-1} \sum_{i=N}^{\infty} \cdots+\sum_{\ell=M}^{\infty} \sum_{i=N}^{\infty} \cdots \\
& \leq \frac{M-1}{\lambda_{1}^{\gamma}} \sum_{i=N}^{\infty}\left(\alpha_{i, \ell}^{n}\right)^{2}+\sum_{l=M}^{\infty} \frac{\varepsilon^{\gamma / m}}{\lambda_{0, l}^{\gamma}} \\
& \leq c \varepsilon^{\gamma / m},
\end{aligned}
$$

for some constant $c>0$. This completes the proof of Claim (3.10).

Since $\left\{\left\|L_{n}\right\|_{1}\right\}_{n \in \mathbb{N}}$ is uniformly bounded with respect to $n \in \mathbb{N}$,

$$
\sum_{i \in \mathbb{N}^{*}}\left|\bar{\nu}_{i}\right|<\infty
$$

For any $\eta \in \mathrm{L}^{2}(\Omega)$, by the Cauchy-Schwarz and the triangle inequality,

$$
\left\|\sum_{i \in \mathbb{N}^{*}}\left\langle\eta, \bar{\psi}_{i}\right\rangle_{\mathrm{L}^{2}(\Omega)} \bar{\nu}_{i} \bar{\psi}_{i}\right\|_{L^{2}(\Omega)} \leq\|\eta\|_{L^{2}(\Omega)} \sum_{i \in \mathbb{N}^{*}}\left|\bar{\nu}_{i}\right|<\infty .
$$


Hence the operator defined through

$$
(\bar{L} \eta)(x)=\sum_{i \in \mathbb{N}^{*}}\left\langle\eta, \bar{\psi}_{i}\right\rangle_{\mathrm{L}^{2}(\Omega)} \bar{\nu}_{i} \bar{\psi}_{i}(x) \quad x \in \Omega, \quad \eta \in \mathrm{L}^{2}(\Omega)
$$

is in $\mathcal{S}_{1}$. Let us prove that $\left\{L_{n}\right\}_{n \in \mathbb{N}}$ converges to $\bar{L}$ in $\mathcal{S}_{1}$. Given $N \in \mathbb{N}^{*}$, denote by $P_{N}^{n}: L^{2}(\Omega) \rightarrow F_{N}^{n}$ the orthogonal projection onto $F_{N}^{n}=\operatorname{span}\left\{\psi_{i}^{n}: 1 \leq i \leq N-1\right\}$ and by $Q_{N}^{n}=I-P_{N}^{n}$ the projection onto $\left(F_{N}^{n}\right)^{\perp}$ :

$\left\|L_{n}-L\right\|_{1} \leq\left\|\left(L_{n}-L\right) P_{N}\right\|_{1}+\left\|L_{n} Q_{N}^{n}\right\|_{1}+\left\|L Q_{N}\right\|_{1}+\left\|L_{n}\left(Q_{N}^{n}-Q_{N}\right)\right\|_{1}$.

The first term converges to zero, because of the strong convergence of the first $N-1$ eigenvalues and eigenfunctions in $\mathbb{R}$ and $\mathrm{L}^{2}(\Omega)$ respectively. From (3.10) we have that the second and third terms are small if $N \in \mathbb{N}^{*}$ is large enough, independent of $n \in \mathbb{N}$, since

$$
\left(\sum_{i \in \mathbb{N}^{*}}\left|\nu_{i}\right|^{n}\right)^{m} \leq \sum_{i \in \mathbb{N}^{*}}\left|\nu_{i}^{n}\right|^{m}<\varepsilon
$$

Using (2.2), we have that

$$
\left\|L_{n}\left(Q_{N}^{n}-Q_{N}\right)\right\|_{1} \leq\left\|L_{n}\right\|_{1} \cdot\left\|Q_{N}^{n}-Q_{N}\right\|
$$

which converges to zero as $n \rightarrow \infty$, since $Q_{N}^{n}-Q_{N}=P_{N}^{n}-P_{N}$ converges to zero for the same reasons as the first term.

Proof of ii) Assume now that $\sup _{n \in \mathbb{N}} \sum_{i \in \mathbb{N}^{*}}\left|\nu_{i}^{n}\right|^{m}=C_{1}$ is finite, so that using the monotonicity of $\left\{\left|\nu_{i}^{n}\right|^{m}\right\}_{i \in \mathbb{N}^{*}}$, for any $m^{\prime}>m$ and any $N \in \mathbb{N}^{*}$,

$$
\sum_{i=N}^{\infty}\left|\nu_{i}^{n}\right|^{m^{\prime}} \leq\left(\nu_{N}^{n}\right)^{m^{\prime}-m} \sum_{i=N}^{\infty}\left|\nu_{i}^{n}\right|^{m} \leq\left|\nu_{N}^{n}\right|^{m^{\prime}-m} C_{1} .
$$

If $\bar{\nu}_{i}=0$ for all $i \in \mathbb{N}^{*}$, then

$$
\lim _{n \rightarrow \infty} \sum_{i \in \mathbb{N}^{*}}\left|\nu_{i}^{n}\right|^{m^{\prime}} \leq \lim _{n \rightarrow \infty}\left|\nu_{N}^{n}\right|^{m^{\prime}-m} C_{1}=0 .
$$

From here on, taking $m^{\prime}=1$ and arguing as before we obtain that $\left\{L_{n}\right\}_{n \in \mathbb{N}}$ converges to 0 in $\mathcal{S}_{1}$. The general case, i.e., when there is $i_{0} \in \mathbb{N}^{*}$ such that $\left|\bar{\nu}_{i_{0}}\right|>0$, follows from similar arguments.

Proof of iii) The convergence of the kernels $K_{L_{n}}$ to the kernel of the limit operator $L$ follows from ii) with $m^{\prime}=1$ and from the strong convergence of $\psi_{i}^{n}$ to $\bar{\psi}_{i}$ in $\mathrm{L}^{2}(\Omega)$.

Remark 3.1. The properties shown in Theorem 3.3 is an analogous at operators level of the compactness of the embedding $\mathrm{H}_{0}^{1}(\Omega) \hookrightarrow \mathrm{L}^{2}(\Omega)$. 


\section{Applications}

In this section we present three applications of the results discussed in this paper. The three cases correspond to minimization problems arising in Quantum Mechanics.

4.1. Minimization of the free energy functional. Consider first the free energy functional

$$
\mathcal{F}_{V, \beta}^{\lambda}(L)=\mathcal{F}_{V, \beta}(L)-\lambda \operatorname{Tr}[L] .
$$

Theorem 4.1. Let $V$ be a potential verifying (3.7) for some $\varepsilon \in(0,1]$ and take $\lambda \leq \lambda_{V, 1}^{\varepsilon}$. Let $F \in \mathcal{C}\left(-\frac{\varepsilon}{2} \Delta, \Omega\right)$ and $\beta$ given by

$$
\beta(s) \equiv F^{*}(-s) \quad \forall s \in \mathbb{R} \text {. }
$$

Then $\left(\beta^{\prime}\right)^{-1}(\Delta-V+\lambda) \equiv L_{\infty} \in \mathcal{H}_{+}^{1}$ is such that

$$
\mathcal{F}_{V, \beta}^{\lambda}\left(L_{\infty}\right)=\inf _{L \in \mathcal{H}_{+}^{1}} \mathcal{F}_{V, \beta}^{\lambda}(L)
$$

provided one of the following conditions is satisfied:

i) if $d=1, V \in \mathrm{L}^{q}(\Omega)$, for some $q \in[1, \infty]$,

ii) if $d=2, V \in \mathrm{L}^{q}(\Omega)$, for some $\left.\left.q \in\right] 1, \infty\right]$,

iii) if $d \geq 3, V \in \mathrm{L}^{q}(\Omega)$, for some $q \in\left[\frac{d}{2}, \infty\right]$,

and $L_{\infty}$ is the unique minimizer of $\mathcal{F}_{V, \beta}^{\lambda}$.

Proof. By Proposition 3.5, the functional $\mathcal{F}_{V, \beta}^{\lambda}$ is bounded from below. Let $\left\{L_{n}\right\}_{n \in \mathbb{N}^{*}} \subset \mathcal{H}_{+}^{1}$ be a minimizing sequence, that is

$$
\lim _{n \rightarrow \infty} \mathcal{F}_{V, \beta}^{\lambda}\left(L_{n}\right)=\inf _{L \in \mathcal{H}_{+}^{1}} \mathcal{F}_{V, \beta}^{\lambda}(L)
$$

Then the sequences

$$
\left\{\left\|L_{n}\right\|_{1}\right\}_{n \in \mathbb{N}^{*}},\left\{\mathcal{K}\left(L_{n}\right)\right\}_{n \in \mathbb{N}^{*}}, \quad\left\{\mathcal{E}_{\beta}\left(L_{n}\right)\right\}_{n \in \mathbb{N}^{*}} \text { and }\left\{\mathcal{P}_{V}\left(L_{n}\right)\right\}_{n \in \mathbb{N}^{*}}
$$

are bounded according to Corollary 3.6. Then Theorem 3.3 provides the existence of $L_{\infty} \in \mathcal{S}_{1}$ such that, up to a subsequence, $\left\{L_{n}\right\}_{n \in \mathbb{N}^{*}}$ converges to $L_{\infty}$ in $\mathcal{S}_{1}$ so that, in particular,

$$
\lim _{n \rightarrow \infty}\left\|L_{n}\right\|_{1}=\left\|L_{\infty}\right\|_{1} .
$$

In order to study the entropy term we consider the space $\ell^{1}$ with the usual norm. Consider the set

$$
\mathcal{A}_{+} \equiv\left\{\mu=\left\{\mu_{i}\right\}_{i \in \mathbb{N}^{*}} \in \ell^{1}: \sum_{i \in \mathbb{N}^{*}} \beta\left(\mu_{i}\right) \geq A\right\}
$$


where $A \equiv \inf _{n \in \mathbb{N}} \mathcal{E}_{\beta}\left(L_{n}\right)$. Both the function $D: \mathcal{A}_{+} \rightarrow \mathbb{R}$ defined by

$$
D(\mu) \equiv \sum_{i \in \mathbb{N}^{*}} \beta\left(\mu_{i}\right) \quad \forall \mu=\left\{\mu_{i}\right\}_{i \in \mathbb{N}^{*}} \in \mathcal{A}_{+},
$$

and the set $\mathcal{A}_{+}$are convex. Thus $D$ is weakly lower semi-continuous, so that $\liminf _{n \rightarrow \infty} D\left(\nu_{n}\right) \geq D\left(\nu_{0}\right)$, where $\nu^{n}=\left\{\nu_{i}^{n}\right\}_{i \in \mathbb{N}^{*}}$ and $\nu_{0}=$ $\left\{\bar{\nu}_{i}\right\}_{i \in \mathbb{N}^{*}}$. This allows to say that

$$
\liminf _{n \rightarrow \infty} \mathcal{E}_{\beta}\left(L_{n}\right) \geq \mathcal{E}_{\beta}\left(L_{\infty}\right) .
$$

Next we consider the kinetic energy term. Given a fixed $N \in \mathbb{N}^{*}$, for any $n \in \mathbb{N}$ we have that

$$
\begin{aligned}
\liminf _{n \rightarrow \infty} \sum_{i \in \mathbb{N}^{*}} \nu_{i}^{n} \int_{\Omega}\left|\nabla \psi_{i}^{n}(x)\right|^{2} d x & \geq \liminf _{n \rightarrow \infty} \sum_{i=1}^{N} \nu_{i}^{n} \int_{\Omega}\left|\nabla \psi_{i}^{n}(x)\right|^{2} d x \\
& \geq \sum_{i=1}^{N} \bar{\nu}_{i} \int_{\Omega}\left|\nabla \bar{\psi}_{i}(x)\right|^{2} d x .
\end{aligned}
$$

Since the number $N$ is arbitrary, we get

$$
\liminf _{n \rightarrow \infty} \mathcal{K}\left(L_{n}\right) \geq \mathcal{K}\left(L_{\infty}\right)
$$

whence $L_{\infty} \in \mathcal{H}_{+}^{1}$. As for the potential energy, we obtain

$$
\lim _{n \rightarrow \infty} \mathcal{P}_{V}\left(L_{n}\right)=\mathcal{P}_{V}\left(L_{\infty}\right)
$$

using Proposition 2.2.

At this point we relate the minimization problem with the one studied in [5]. For this purpose we denote by $S$ the set of non-increasing sequences $\left\{\nu_{i}\right\}_{i \in \mathbb{N}^{*}} \subset \mathbb{R}_{+}$converging to zero, such that $\sum_{i \in \mathbb{N}^{*}} \beta\left(\nu_{i}\right)$ is absolutely convergent and let

$$
\mathcal{X} \equiv\left\{(\nu, \psi) \in S \times\left(\mathrm{L}^{2}(\Omega)\right)^{\mathbb{N}^{*}}:\left\langle\psi_{i}, \psi_{j}\right\rangle_{\mathrm{L}^{2}(\Omega)}=\delta_{i j}, \forall i, j \in \mathbb{N}^{*}\right\}
$$

be the space of mixed states. Then we define an associated free energy functional acting on mixed states as

$$
\mathcal{F}_{V, \beta}^{\lambda}[\nu, \psi] \equiv \sum_{i \in \mathbb{N}^{*}}\left[\beta\left(\nu_{i}\right)+\nu_{i} \int_{\Omega}\left(\left|\nabla \psi_{i}\right|^{2}+(V(x)-\lambda)\left|\psi_{i}\right|^{2}\right) d x\right] .
$$

Next we assume some extra hypotheses on $\beta$ in order to be in the context of Section 3 in [5]. We assume that $\beta$ is of class $C^{1}$ and strictly convex. We also assume that $-\Delta+V$ has an infinite sequence $\left\{\lambda_{i}(V)\right\}_{i \in \mathbb{N}^{*}}$ of eigenvalues diverging to $\infty$. This last assumption is always true when $\Omega$ is bounded. 
We observe that the function $F$ is simply given by

$$
F(s)=\beta \circ\left(\beta^{\prime}\right)^{-1}(-s)-s\left(\beta^{\prime}\right)^{-1}(-s) .
$$

As a consequence of Theorem 4.1,

$$
\min _{(\nu, \psi) \in \mathcal{X}} \mathcal{F}_{V, \beta}^{\lambda}[\nu, \psi]
$$

has a solution $(\nu, \psi) \in \mathcal{X}$ given by $\nu_{i}=\nu_{i}\left(L_{\infty}\right)$ and $\psi_{i}=\psi_{i}\left(L_{\infty}\right)$.

The minimizer of (4.1) is unique, up to the choice of basis for nonsimple eigenvalues, as proved in [5]. As a consequence the minimization problem at the level of operators

$$
\min _{L \in \mathcal{H}_{+}^{1}} \mathcal{F}_{V, \beta}^{\lambda}(L)
$$

has a unique minimizer $L_{\infty} \in \mathcal{H}_{+}^{1}$. The solution of (4.1) is given by

$$
(\bar{\nu}, \bar{\psi})=\left\{\left(\bar{\nu}_{i}, \bar{\psi}_{i}\right)\right\}_{i \in \mathbb{N}^{*}} \in \mathcal{X},
$$

where

$$
\bar{\nu}_{i}=\left(\beta^{\prime}\right)^{-1}\left(\lambda-\lambda_{V, i}\right) \text {, }
$$

and $\bar{\psi}_{i}$ is an eigenfunction of $-\Delta+V-\lambda$ associated to $\lambda_{V, i}$. Finally we may simply rewrite this as

$$
L_{\infty}=\left(\beta^{\prime}\right)^{-1}(\Delta-V+\lambda) .
$$

Remark 4.1. In the Heisenberg formalism we see that the solution to the minimization problem given by Theorem 4.1 is a stationary solution to the Heisenberg equation

$$
\left[-\Delta+V-\lambda, L_{\lambda}\right]=0
$$

Recall that the commutator operator is given by $[L, R]=L R-R L$.

4.2. Free energy involving a non-linear but local function of the density function. Consider the free energy functional given by

$$
\mathcal{F}_{V, \beta}^{\lambda, g}(L) \equiv \mathcal{F}_{V, \beta}^{\lambda}+\mathcal{G}(L) \quad \forall L \in \mathcal{H}_{+}^{1},
$$

where

$$
\mathcal{G}(L)=\int_{\Omega} g\left(\rho_{L}(x)\right) d x
$$

and $g$ is some real function, which is not necessarily convex. Using an argument similar to that in the proof of Theorem 4.1, we obtain the following result. 
Theorem 4.2. Let $V$ be a potential on $\Omega$ verifying (3.7), for some $\varepsilon \in(0,1]$. Let $\lambda \leq \lambda_{V, 1}^{\varepsilon}$ and assume that $F \in \mathcal{C}\left(-\frac{\varepsilon}{2} \Delta, \Omega\right)$ and $\beta$ is given by

$$
\beta(s) \equiv F^{*}(-s) \quad \forall s \in \mathbb{R} .
$$

Let $g \in \mathrm{C}([0, \infty))$ be such that for non-negative constants $c_{1}, c_{2}$

$$
c_{1} \leq g(s) \leq c_{2} s^{q} \quad \forall s \geq 0,
$$

where

i) $q \in[1, \infty)$ if $d=1$ or $d=2$,

ii) $q \in[1, d /(d-2)]$ if $d \geq 3$.

Then there exists $L_{\infty} \in \mathcal{H}_{+}^{1}$ such that

$$
\mathcal{F}_{V, \beta}^{\lambda, g}\left(L_{\infty}\right)=\inf _{L \in \mathcal{H}_{+}^{1}} \mathcal{F}_{V, \beta}^{\lambda, g}(L) .
$$

Proof. It is similar to the one of Theorem 4.1. We use condition (4.2) to show via Fatou's lemma that

$$
\mathcal{G}\left(L_{\infty}\right) \leq \liminf _{n \rightarrow \infty} \mathcal{G}\left(L_{n}\right)
$$

where $\left\{L_{n}\right\}_{n \in \mathbb{N}^{*}} \subset \mathcal{H}_{+}^{1}$ is a minimizing sequence for $\mathcal{F}_{V, \beta}^{\lambda, g}$.

Remark 4.2. If $g \in \mathrm{C}^{1}([0, \infty)), L_{\infty}$ is a fixed point of the application $Y: \mathcal{H}_{+}^{1} \longrightarrow \mathcal{H}_{+}^{1}$ given by

$$
Y(L)=\left(\beta^{\prime}\right)^{-1}\left(-(-\Delta+V)+\lambda-g^{\prime} \circ \rho_{L}\right) .
$$

4.3. Stationary states for the Hartree problem with temperature. Consider a Heisenberg equation with a Poisson coupling, namely

$$
\begin{cases}i \partial_{t} L(t)=[-\Delta+V(t, \cdot), L(t)] & t \geq 0, \\ -\Delta V(t, x)=\rho_{L(t)}(x) & x \in \Omega, \quad t \geq 0 \\ L(0)=\tilde{L} & \end{cases}
$$

where $L(t)$, the density operator of the system, is a positive trace-class operator acting on $\mathrm{L}^{2}(\Omega)$ and $[L, R]=L R-R L$. This system is known as the Hartree evolution system, or Schrödinger-Poisson system in the mixed states formulation, and a large literature has been devoted to its study, which goes far beyond the scope of this paper. We refer to [18] for further references.

We assume that $d \leq 4$ and restrict our study to the case of homogeneous Dirichlet boundary conditions:

$$
V=0 \quad \text { on } \partial \Omega
$$


The stationary states of (4.3) are then solutions of

$$
\left\{\begin{array}{l}
{[-\Delta+V, L]=0,} \\
-\Delta V=\rho_{L} .
\end{array}\right.
$$

Stationary states of (4.3) can be obtained through the minimization of the free energy

$$
\mathcal{F}_{\beta}(L)=\mathcal{E}_{\beta}(L)+\mathcal{K}(L)+\mathcal{P}(L) \quad \forall L \in \mathcal{H}_{+}^{1},
$$

where

$$
\mathcal{P}(L)=\frac{1}{2} \int_{\Omega} V_{L} \rho_{L} d x=\frac{1}{2} \int_{\Omega}\left|\nabla V_{L}\right|^{2} d x .
$$

Theorem 4.3. Let $F \in \mathcal{C}(-\Delta, \Omega)$ and

$$
\beta(s) \equiv F^{*}(-s), \quad \forall s \in \mathbb{R} .
$$

Then there exists $L_{F} \in \mathcal{H}_{+}^{1}$ such that

$$
\mathcal{F}_{\beta}\left(L_{F}\right) \leq \mathcal{F}_{\beta}(L) \quad \forall L \in \mathcal{H}_{+}^{1} .
$$

Moreover if $\beta$ is of class $C^{1}$ in the interior of its support, then

$$
L_{F}=\left(\beta^{\prime}\right)^{-1}\left(\Delta-V_{L_{F}}\right)
$$

is the unique minimizer of $\mathcal{F}_{\beta}$ and solves (4.4) as well.

Proof. The proof follows the same lines as the one for Theorem 4.1. The argument changes only to reach $\lim _{n \rightarrow \infty} \mathcal{P}\left(L_{n}\right)=\mathcal{P}\left(L_{F}\right)$, but this still follows from Proposition 2.2.

Remark 4.3. Let us notice that if $\beta$ is non-negative then the minimizer in Theorem 4.3 is $L_{F}=0$. However, the result applies to functions $\beta$ for which $\{\beta<0\} \neq \emptyset$ as it is the case for

$$
\beta(s)= \begin{cases}s \log s-s & \text { if } s>0 \\ 0 & \text { if } s=0 \\ +\infty & \text { if } s<0\end{cases}
$$

The result is still interesting for practical cases for which $\beta^{\prime}$ is continuous at $0_{+}$. Replacing $\beta$ by $\beta-\lambda$ for some positive $\lambda$, we can produce non-trivial solutions. The parameter $\lambda$ in this case can be reinterpreted as a Lagrange multiplier (see below).

Remark 4.4. The case with an attracting Poisson coupling, that is when the potential is given by

$$
+\Delta V=\rho_{L} \quad \text { in } \Omega,
$$


can be dealt with the same methods although it makes less sense from the point of view of physics. Some additional work is necessary to establish spectral properties of $\Delta+V_{L}$.

Let us finally remark that for stationary states having a prescribed total charge we may get a generalization of [18, Theorem 2] at operators level. Mathematically, the free energy is changed only by a term $-\lambda \int_{\Omega} \rho_{L} d x$, where $\lambda$ is the Lagrange multiplier associated to the mass constraint. The generating function $\beta$ of the entropy term is now changed into $\nu \mapsto \beta(\nu)-\lambda \nu$, which results in the fact that the set $\{\nu \in \mathbb{R}: \nu \mapsto \beta(\nu)-\lambda \nu<0\}$ is automatically non-empty if $\beta^{\prime}$ is continuous at $0_{+}$. Because of the compactness property, the mass constraint will be verified when passing to the limit the minimizing sequence.

Moreover, with almost no work, we may add an external potential which takes negative values and eventually singularities, of Coulomb type, for instance. This situation is highly relevant from a physics point of view, for the modelization of atomic and molecular systems, without temperature, see for instance [23] and references therein, or with temperature, see [17]. In such a case however, the appropriate model is rather the Hartree-Fock system than the Hartree system.

Acknowledgments: The authors would like to thank Eric Paturel for some fruitful discussions about the operator setting for this work.

This work has been supported by ECOS-Conicyt under grants \# C02E08 and C05E09. J.D., P.F. and J.M. respectively acknowledge partial support from the European Programs HPRN-CT \# 2002-00277 \& 00282, Fondecyt Grant \# 1030929 and FONDAP Matemáticas Aplicadas, and Proyecto MECESUP UCH0009 and the European Alfa program.

(C) 2006 by the authors. This paper may be reproduced, in its entirety, for noncommercial purposes.

\section{REFERENCES}

[1] N. Ben Abdallah and J. Dolbeault, Relative entropies for kinetic equations in bounded domains (irreversibility, stationary solutions, uniqueness), Arch. Ration. Mech. Anal., 168 (2003), pp. 253-298.

[2] R. D. Benguria And M. Loss, Connection between the Lieb-Thirring conjecture for Schrödinger operators and an isoperimetric problem for ovals on the plane, in Partial differential equations and inverse problems, vol. 362 of Contemp. Math., Amer. Math. Soc., Providence, RI, 2004, pp. 53-61.

[3] H. Brezis, Analyse fonctionnelle, Collection Mathématiques Appliquées pour la Maîtrise., Masson, Paris, 1983. 
[4] J. A. Carrillo, A. Jüngel, P. A. Markowich, G. Toscani, and A. UnTERREITER, Entropy dissipation methods for degenerate parabolic problems and generalized Sobolev inequalities, Monatsh. Math., 133 (2001), pp. 1-82.

[5] J. Dolbeault, P. Felmer, M. Loss, and E. Paturel, Lieb-Thirring type inequalities and Gagliardo-Nirenberg inequalities for systems, J. Functional Analysis, (2006).

[6] J. Dolbeault, P. Markowich, D. Ölz, and C. Schmeiser, Nonlinear diffusions as limit of kinetic equations with relaxation collision kernels, To appear, (2006).

[7] J. Dolbeault, P. A. Markowich, and A. Unterreiter, On singular limits of mean-field equations, Arch. Ration. Mech. Anal., 158 (2001), pp. 319351.

[8] A. Eden and C. Foias, A simple proof of the generalized Lieb-Thirring inequalities in one-space dimension, J. Math. Anal. Appl., 162 (1991), pp. 250254.

[9] J.-M. Ghidaglia, M. Marion, and R. Temam, Generalization of the Sobolev-Lieb-Thirring inequalities and applications to the dimension of attractors, Differential Integral Equations, 1 (1988), pp. 1-21.

[10] D. Gilbarg And N. S. Trudinger, Elliptic partial differential equations of second order, vol. 224 of Grundlehren der Mathematischen Wissenschaften [Fundamental Principles of Mathematical Sciences], Springer-Verlag, Berlin, second ed., 1983.

[11] V. Glaser and A. Martin, Comment on the paper: "Necessary conditions on potential functions for nonrelativistic bound states" by G. Rosen, Lett. Nuovo Cimento (2), 36 (1983), pp. 519-520.

[12] Y. Guo And G. Rein, Stable steady states in stellar dynamics, Arch. Ration. Mech. Anal., 147 (1999), pp. 225-243.

[13] —, Isotropic steady states in galactic dynamics, Comm. Math. Phys., 219 (2001), pp. 607-629.

[14] E. Lieb And W. Thirring, E. Lieb, B. Simon, A. Wightman Eds., Princeton University Press, 1976, ch. Inequalities for the moments of the eigenvalues of the Schrödinger Hamiltonian and their relation to Sobolev inequalities, pp. 269-303.

[15] E. H. LiEB, Kinetic energy bounds and their application to the stability of matter, in Schrödinger operators (Sønderborg, 1988), vol. 345 of Lecture Notes in Phys., Springer, Berlin, 1989, pp. 371-382.

[16] — Bounds on Schrödinger operators and generalized Sobolev-type inequalities with applications in mathematics and physics, in Inequalities (Birmingham, 1987), vol. 129 of Lecture Notes in Pure and Appl. Math., Dekker, New York, 1991, pp. 123-133.

[17] P.-L. Lions, Hartree-Fock and related equations, in Nonlinear partial differential equations and their applications. Collège de France Seminar, Vol. IX (Paris, 1985-1986), vol. 181 of Pitman Res. Notes Math. Ser., Longman Sci. Tech., Harlow, 1988, pp. 304-333.

[18] P. Markowich, G. Rein, and G. Wolansky, Existence and nonlinear stability of stationary states of the Schrödinger-Poisson system, J. Statist. Phys., 106 (2002), pp. 1221-1239. 
[19] M. Reed And B. Simon, Methods of modern mathematical physics. I. Functional analysis, Academic Press, New York, 1972.

[20] — Methods of modern mathematical physics. II. Fourier analysis, selfadjointness, Academic Press [Harcourt Brace Jovanovich Publishers], New York, 1975.

[21] G. ReIN, Non-linear stability of gaseous stars, Arch. Ration. Mech. Anal., 168 (2003), pp. 115-130.

[22] G. Rosen, Necessary conditions on potential functions for nonrelativistic bound states, Phys. Rev. Lett., 49 (1982), pp. 1885-1887.

[23] J. P. SoloveJ, The ionization conjecture in Hartree-Fock theory, Ann. of Math. (2), 158 (2003), pp. 509-576.

[24] W. Thirring, A course in mathematical physics. Vol. 3, Springer-Verlag, New York, 1981. Quantum mechanics of atoms and molecules, Translated from the German by Evans M. Harrell, Lecture Notes in Physics, 141.

[25] E. J. M. Veling, Lower bounds for the infimum of the spectrum of the Schrödinger operator in $\mathbb{R}^{N}$ and the Sobolev inequalities, JIPAM. J. Inequal. Pure Appl. Math., 3 (2002), pp. Article 63, 22 pp. (electronic). Corrigendum: 5, Article 109, 2 pp. (electronic).

[26] M. I. Weinstein, Nonlinear Schrödinger equations and sharp interpolation estimates, Comm. Math. Phys., 87 (1982/83), pp. 567-576.

[27] H. WeYL, Das asymptotische Verteilungsgesetz der Eigenwerte linearer partieller Differentialgleichungen (mit einer Anwendung auf die Theorie der Hohlraumstrahlung), Math. Ann., 71 (1912), pp. 441-479.

[28] G. Wolansky and M. Ghil, An extension of Arnol'd's second stability theorem for the Euler equations, Phys. D, 94 (1996), pp. 161-167.

Jean Dolbeault

Ceremade (UMR CNRS no. 7534) — Université Paris Dauphine

Place de Lattre de Tassigny, 75775 Paris CÉdeX 16, France

E-mail address: dolbeaul@ceremade.dauphine.fr

PATRicio FELMER

Departamento de Ingeniería Matemática - Universidad de Chile Blanco Encalada 2120 (5TO Piso), Santiago, Chile

E-mail address: pfelmer@dim.uchile.cl

JuAN Mayorga-Zambrano

Departamento de Ingeniería Matemática - Universidad de Chile Blanco Encalada 2120 (4to Piso),

Santiago, Chile

E-mail address: jmayorga@dim.uchile.cl 